\title{
Stochastic Modeling in Stock Market Trend Prediction
}

\author{
N. Sonai Muthu", K. Senthamarai Kannan*, K.M. Karuppasamy ${ }^{\#}$ and V. Deneshkumar ${ }^{+}$ \\ Department of Statistics, Manonmaniam Sundaranar University, Abishekapatti, Tirunelveli - 627012 , \\ Tamil Nadu, India. \\ sonaimuthu@gmail.com ${ }^{\$}$, senkannan2002@gmail.com ${ }^{*}$, m.k.samystat@gmail.com ${ }^{\#}$ and vdenesh77@gmail.com ${ }^{+}$
}

\begin{abstract}
:-
In Modern centuries a lot of predicting techniques take been proposed and applied for the stock market movement prediction. In this paper, the pattern examinations of the financial exchange forecast are introduced by utilizing Hidden Markov Model with the one day distinction in close incentive for a particular period. The likelihood esteems $\pi$ gives the pattern level of the stock costs which is determined for all the notice arrangement and stowed away successions. It supports for decision makers to make decisions in case of indecision on the basis of the proportion of probability values found from the steady state probability distribution.
\end{abstract}

Keywords: Markov chain, Transition Probability Matrix, Hidden Markov Model and Stock Market

\section{Introduction}

The most two popular trending in Indian stock market classified named as the Bombay Stock Exchange (BSE) and the National Stock Exchange (NSE). The BSE has been started since 1875. The NSE has been founded in 1992 and started trading in 1994. Even though both exchanges following the same trading mechanism, trading hours, settlement process, etc. The two prominent Indian market indexes are Sensex and S\&P CNX Nifty.

Financial market (Stock Market) is a platform for shareholders to particular some bonds of a company. Investors will become a part of the company members and share in both gain and losses of that particular company. This is the better way for the investors to get extra income apart from their permanent jobs. Variations of share prices on every day make it more volatile and difficult to forecast the upcoming price. When purchasing a stock, it does not any guarantees are anything in return. Thus, it makes stocks risky in investment, but investors can get high profit return. When investors are taking wrong decision in choosing the counters, it may end up in capital loss. The behavior of financial market revenues has been deeply discoursed over some years.

Hassan and Nath (2005) utilized HMM to anticipate following day closing price for a portion of the airplanes. Gupta and Dhingra (2012), have carried out HMM by utilizing different perception information (open, close, low, exorbitant costs) of stock to forecast its closing prices. Jyoti Badge (2012) used 
methodological pointers as an input variable instead of stock prices used for investigation. Kavitha (2013), had applied HMMs for one day close value of a particular period for stock market trend analysis using BaumWelch algorithm. They used MATLAB to get hidden states sequence. Therefore, the investors can observe easily the behavioral pattern of the stock market. Sasikumar and Sheikh Abdullah (2015), an attempt to various stochastic modeling using BSE Oil gas Stock data set. Sasikumar and Sheikh Abdullah (2016), an attempt to prediction of financial market (Stock Market) using hidden markov model and also making one day changes in close value of stock market value has been used for some period and the parallel transition probability matrix and emission probability matrix are obtained.

Mohamed Ashik and Senthamarai Kannan (2017), applied to ARIMA model to forecast in NSE closing stock price. Nguyet Nguyen (2018), have established the hidden Markov model to forecast once-amonth closing prices of the S\&P 500 and formerly used these estimates to trade the stock using the four measures: AIC, BIC, HQC, and CAIC to inspect the concerts of HMM with numbers of states from two to six. Ali Sasani and Stephanie Tibado (2020), have used to machine learning techniques on stock market prediction. Deneshkumar et al., (2020), have used to hidden Markov model in trend analyses of the stock market prediction are presented.

The trend analysis of the stock market is discovered utilizing Hidden Markov Model by considering the one day distinction in close incentive for a specific period. For an offered perspective succession, the hidden sequence of states and their relating likelihood esteems are found. The probability values of $\pi$ gives the trend level of the stock costs. Leaders present choices in defense of helplessness. The proposed approach gives a stage for leaders to settle on choices based on the level of likelihood esteems acquired from the consistent state likelihood dissemination. The hidden states and grouping are created for financial exchange information utilizing Hidden Markov model through MATLAB programming.

This paper is prepared into five sections as follows, Section II Basic concepts and definition of Markov chain and Transition Probability matrix. Section III Explaining and Construction of Hidden Markov Model, Section IV Deals the numerical results and discussion of proposed method. Finally, Conclusions in Section V.

\section{Markov Chain}

A Markov chain is a discrete random process with the property that the next state depends only on the current state; the past states have no influence on the future. The Stochastic process $X=\left\{X_{n}, n=0,1,2,3, \ldots\right\}$ is called a Markov Chain (MC) provided that 


$$
\begin{aligned}
& P_{r}=P_{r}\left\{X_{n+1}=j \mid X_{n}=i, X_{n-1}=i_{n-1}, \ldots, X_{1}=i_{1}, X_{0}=i_{0}\right\} \\
& P_{r}=P_{r}\left\{X_{n+1}=j \mid X_{n}=i\right\}, \text { for all } \mathrm{j} \in S \quad \ldots(1)
\end{aligned}
$$

where, $n$ is independent and $S$ is the countable state space. The probabilities $P_{i j}$ are then called the transition probabilities for the Markov chain $X$.

\subsection{Transition Probability Matrix}

The transition probability $\mathrm{P}_{\mathrm{jk}}$ satisfy $\mathrm{P}_{\mathrm{jk}} \geq 0, \sum_{k} P_{j k}=1$ for all $\mathrm{j}$. These probabilities might be written in the framework structure.

$$
P=\left[\begin{array}{cccc}
p_{11} & p_{12} & p_{13} & \ldots \\
p_{21} & p_{22} & p_{23} & \ldots \\
\cdots & \cdots & \cdots & \ldots \\
\cdots & \cdots & \ldots & \cdots
\end{array}\right]
$$

This is called the transition probability matrix (TPM). P is a stochastic matrix ie., a square matrix with non-negative elements and row total is equal to one.

\section{Construction of Hidden Markov Model}

Hidden Markov model is very effective and intuitive approach to many sequential pattern such as thermodynamics, statistical mechanics, protein sequence analysis, multiple sequence alignments, physics, chemistry, economics, finance, signal processing, information theory, gene annotation, classification, similarity search.

Hidden Markov model (HMM) is a stochastic model which isn't straightforwardly recognizable, it depicts the discernible events that are depend on upon inside factors. Deneshkumar et al., (2020), the observable events are represented as symbols, where the invisible factor involved in the observation is represented as a state. Kavitha et al., (2013), HMM is a stochastic model where the system is assumed to be a Markov Process with hidden states. HMM gives better accuracy than other models. Utilizing the given information esteems, the boundaries of the $\operatorname{HMM}(\lambda)$ meant by A, B and $\pi$ are discovered.

\section{HMM consists of}

A set of hidden or latent states (S); A set of possible output symbols (O); A state transition probability matrix (A)Probability of making transition from one state to each of the other states observation emission probability matrix (B). Probability of emitting/observing a symbol at a particular state. Prior probability matrix $(\pi)$. 
Probability of starting at a particular state: $\mathrm{An} \mathrm{HMM}$ is defined as $\lambda=(\mathrm{S}, \mathrm{O}, \mathrm{A}, \mathrm{B}, \pi)$. where $\mathrm{S}=\{\mathrm{S} 1, \mathrm{~S} 2, \ldots, \mathrm{SN}\}$ is a set of $\mathrm{N}$ possible states and $\mathrm{O}=\{\mathrm{O} 1, \mathrm{O} 2, \ldots, \mathrm{On}\}$ is a set of $\mathrm{M}$ possible observation symbols, $\mathrm{A}$ is an $\mathrm{N} \times \mathrm{N}$ state Transition Probability Matrix (TPM), B is an $\mathrm{N} \times \mathrm{M}$ observation or Emission Probability Matrix (EPM) and $\pi$ is an N dimensional initial state probability distribution vector and A,B and $\pi$ should satisfy the following conditions.

$$
\begin{array}{ll}
\sum_{j=1}^{N} a_{i j}=1 & \text { where } 1 \leq i \leq N ; \\
\sum_{j=1}^{M} b_{i j}=1 & \text { where } 1 \leq i \leq M ; \\
\sum_{i=1}^{N} \pi_{i}=1 & \text { where } \pi_{i} \geq 0
\end{array}
$$

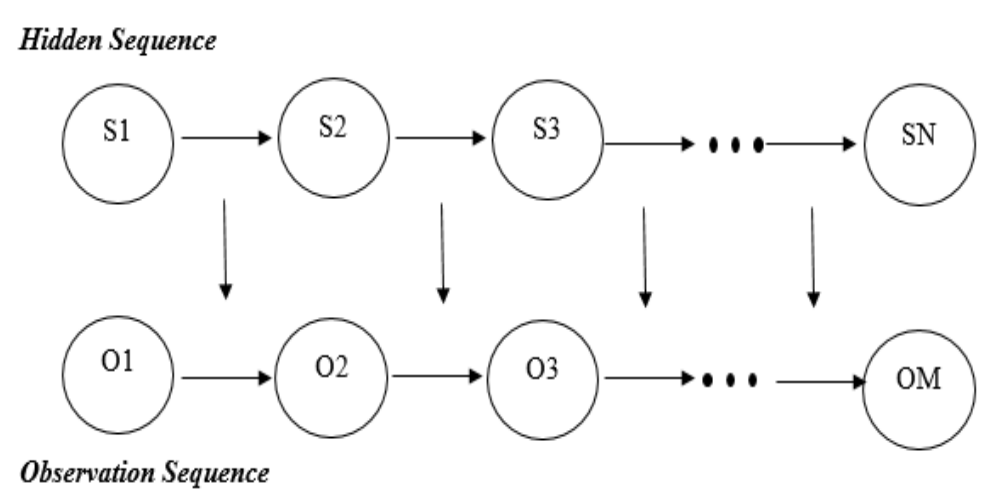

Figure 1: General structure of a hidden Markov model

The main problems of hidden Markov model are: Evaluation, Decoding and Learning.

Evaluation problem - Given the HMM $\lambda=\{A, B, \pi\}$ and the observation sequence $O=O 1, O 2, \ldots, O M$, the probability that model $\lambda$ has generated sequence $O$ is calculated. Regularly this issue is established by the Forward Backward Algorithm (Rabiner, 1989 \& 1993).

Decoding problem - Given the $\mathrm{HMM} \lambda=\{\mathrm{A}, \mathrm{B}, \pi\}$ and the observation sequence $\mathrm{O}=\mathrm{O} 1, \mathrm{O} 2, \ldots, \mathrm{OM}$, calculate the most likely sequence of hidden states that produced this observation sequence O. Generally this issue is dealt with by Viterbi Algorithm (Rabiner,1989 \& 1993)

Learning problem - Given some training observation sequences $\mathrm{O}=\mathrm{O} 1, \mathrm{O} 2,, \ldots, \mathrm{OM}$ and general structure of HMM (numbers of hidden and visible states), determine HMM parameters $\lambda=\{A, B, \pi\}$ that best fit 
training data. The most common solution for this problem is Baum - Welch algorithm (Rabiner 1989 \& 1993) which is considered as the traditional method for training HMM.

\section{Results and discussions}

In this study is based on secondary data has been taken from Yahoofinance.com and BPCL.NS, HINDPETRO.NS and IOC.NS and the daily close value data from the month of January 1, 2021 to September 20, 2021 period was considered. The table 1. Shows that the descriptive measures of stock price closing values for ranges between 87.00 Currency in INR to 498.100 Currency in INR. The mean and standard deviation of BPCL.NS closing stock price is 444.225 and 27.7173 which is high among the other two stock values and lowest closing stock price value is IOC.NS. The value of skewness indicates that the closing stock price could be positively/negatively skewed distribution depending upon on the respective stocks.

Table 1: Descriptive Statistics for BPCL.NS, HINDPETRO.NS and IOC.NS Stock Price

\begin{tabular}{|c|l|l|l|l|}
\hline SI. No & \multicolumn{1}{|c|}{ Descriptive } & BPCL.NS & HINDPETRO.NS & \multicolumn{1}{|c|}{ IOC.NS } \\
\hline 1 & Sum & 78627.910 & 45280.650 & 18124.360 \\
\hline 2 & Mean & 444.225 & 255.822 & 102.397 \\
\hline 3 & Median & 449.250 & 252.450 & 103.200 \\
\hline 4 & Mode & 387.300 & 224.900 & 104.650 \\
\hline 5 & Standard Deviation & 27.7173 & 25.5036 & 8.01682 \\
\hline 6 & Variance & 768.254 & 650.435 & 64.270 \\
\hline 7 & Maximum & 498.100 & 309.000 & 118.350 \\
\hline 8 & Minimum & 380.600 & 216.650 & 87.00 \\
\hline 9 & Coeff. of Skewness & -.303 & .392 & -.052 \\
\hline 10 & Coeff. of Kurtosis & -.752 & -1.029 & -.960 \\
\hline
\end{tabular}

Table 2: The closing value of stock Price for BPCL.NS, HINDPETRO.NS and IOC.NS

\begin{tabular}{|c|c|c|c|c|c|c|c|c|c|c|}
\hline & \multicolumn{4}{|c|}{ BPCL.NS } & \multicolumn{3}{c|}{ HINDPETRO.NS } & \multicolumn{3}{c|}{ IOC.NS } \\
\cline { 2 - 12 } SI. No & Date & $\begin{array}{c}\text { Close } \\
\text { Value }\end{array}$ & $\begin{array}{c}\text { Differ } \\
\text { ence }\end{array}$ & symbol & $\begin{array}{c}\text { Close } \\
\text { Value }\end{array}$ & $\begin{array}{c}\text { Differ } \\
\text { ence }\end{array}$ & symbol & $\begin{array}{c}\text { Close } \\
\text { Value }\end{array}$ & $\begin{array}{c}\text { Differ } \\
\text { ence }\end{array}$ & symbol \\
\hline 1 & $01-01-21$ & 381.95 & - & - & 221.25 & - & - & 91.50 & - & - \\
\hline 2 & $04-01-21$ & 395.35 & 13.40 & I & 222.75 & 1.50 & I & 93.85 & 2.35 & I \\
\hline 3 & $05-01-21$ & 392.00 & -3.35 & D & 223.1 & 0.35 & I & 93.00 & -0.85 & D \\
\hline 4 & $06-01-21$ & 387.30 & -4.70 & D & 220.95 & -2.15 & D & 94.10 & 1.10 & I \\
\hline 5 & $07-01-21$ & 392.20 & 4.90 & I & 222.8 & 1.85 & I & 94.50 & 0.40 & I \\
\hline 6 & $08-01-21$ & 400.15 & 7.95 & I & 225.5 & 2.70 & I & 96.15 & 1.65 & I \\
\hline 7 & $11-01-21$ & 401.75 & 1.60 & I & 224.35 & -1.15 & D & 96.00 & -0.15 & D \\
\hline
\end{tabular}




\begin{tabular}{|c|c|c|c|c|c|c|c|c|c|c|}
\hline 8 & $12-01-21$ & 402.55 & 0.80 & I & 224.9 & 0.55 & I & 97.95 & 1.95 & I \\
\hline 9 & $13-01-21$ & 411.90 & 9.35 & I & 227.15 & 2.25 & I & 100.95 & 3.00 & $\mathrm{I}$ \\
\hline 10 & $14-01-21$ & 426.70 & 14.80 & I & 233.6 & 6.45 & I & 103.20 & 2.25 & I \\
\hline 11 & $15-01-21$ & 414.60 & -12.10 & $\mathrm{D}$ & 228.6 & -5.00 & $\mathrm{D}$ & 101.30 & -1.90 & $\mathrm{D}$ \\
\hline 12 & $18-01-21$ & 409.55 & -5.05 & $\mathrm{D}$ & 231.25 & 2.65 & I & 98.30 & -3.00 & $\mathrm{D}$ \\
\hline 13 & $19-01-21$ & 413.45 & 3.90 & I & 231.9 & 0.65 & $\mathrm{I}$ & 98.55 & 0.25 & $\mathrm{I}$ \\
\hline 14 & $20-01-21$ & 413.85 & 0.40 & I & 231.05 & -0.85 & $\mathrm{D}$ & 98.30 & -0.25 & $\mathrm{D}$ \\
\hline 15 & $21-01-21$ & 408.30 & -5.55 & $\mathrm{D}$ & 228.8 & -2.25 & $\mathrm{D}$ & 96.70 & -1.60 & $\mathrm{D}$ \\
\hline 16 & $22-01-21$ & 396.05 & -12.25 & $\mathrm{D}$ & 225.4 & -3.40 & $\mathrm{D}$ & 95.70 & -1.00 & $\mathrm{D}$ \\
\hline 17 & $25-01-21$ & 387.30 & -8.75 & $\mathrm{D}$ & 220.2 & -5.20 & $\mathrm{D}$ & 93.45 & -2.25 & $\mathrm{D}$ \\
\hline 18 & $27-01-21$ & 380.60 & -6.70 & $\mathrm{D}$ & 216.65 & -3.55 & $\mathrm{D}$ & 91.40 & -2.05 & $\mathrm{D}$ \\
\hline 19 & $28-01-21$ & 384.30 & 3.70 & I & 221.5 & 4.85 & I & 92.60 & 1.20 & I \\
\hline 20 & $29-01-21$ & 383.60 & -0.70 & $\mathrm{D}$ & 218.6 & -2.90 & $\mathrm{D}$ & 93.25 & 0.65 & I \\
\hline 21 & $01-02-21$ & 393.00 & 9.40 & I & 217.95 & -0.65 & $\mathrm{D}$ & 95.80 & 2.55 & I \\
\hline 22 & $02-02-21$ & 408.00 & 15.00 & I & 222.3 & 4.35 & I & 98.80 & 3.00 & I \\
\hline 23 & $03-02-21$ & 414.90 & 6.90 & I & 225.25 & 2.95 & I & 101.85 & 3.05 & I \\
\hline 24 & $04-02-21$ & 418.55 & 3.65 & I & 229.6 & 4.35 & I & 103.60 & 1.75 & I \\
\hline 25 & $05-02-21$ & 415.00 & -3.55 & $\mathrm{D}$ & 225.05 & -4.55 & $\mathrm{D}$ & 102.40 & -1.20 & $\mathrm{D}$ \\
\hline 26 & $08-02-21$ & 420.05 & 5.05 & I & 229.55 & 4.50 & I & 104.10 & 1.70 & I \\
\hline 27 & $09-02-21$ & 419.35 & -0.70 & $\mathrm{D}$ & 226.55 & -3.00 & $\mathrm{D}$ & 97.70 & -6.40 & $\mathrm{D}$ \\
\hline 28 & $10-02-21$ & 415.80 & -3.55 & $\mathrm{D}$ & 224.9 & -1.65 & $\mathrm{D}$ & 96.75 & -0.95 & $\mathrm{D}$ \\
\hline 29 & $11-02-21$ & 421.95 & 6.15 & I & 227.4 & 2.50 & I & 96.50 & -0.25 & $\mathrm{D}$ \\
\hline 30 & $12-02-21$ & 418.10 & -3.85 & $\mathrm{D}$ & 223.85 & -3.55 & $\mathrm{D}$ & 95.10 & -1.40 & $\mathrm{D}$ \\
\hline 31 & $15-02-21$ & 419.00 & 0.90 & I & 222.6 & -1.25 & $\mathrm{D}$ & 94.15 & -0.95 & $\mathrm{D}$ \\
\hline 32 & $16-02-21$ & 417.95 & -1.05 & $\mathrm{D}$ & 225.65 & 3.05 & I & 95.60 & 1.45 & I \\
\hline 33 & $17-02-21$ & 411.70 & -6.25 & $\mathrm{D}$ & 234.45 & 8.80 & I & 95.00 & -0.60 & $\mathrm{D}$ \\
\hline 34 & $18-02-21$ & 432.20 & 20.50 & I & 245.7 & 11.25 & I & 99.00 & 4.00 & I \\
\hline 35 & $19-02-21$ & 430.00 & -2.20 & $\mathrm{D}$ & 244 & -1.70 & $\mathrm{D}$ & 97.55 & -1.45 & $\mathrm{D}$ \\
\hline 36 & $22-02-21$ & 422.55 & -7.45 & $\mathrm{D}$ & 241.5 & -2.50 & $\mathrm{D}$ & 95.65 & -1.90 & $\mathrm{D}$ \\
\hline 37 & $23-02-21$ & 435.25 & 12.70 & I & 244.9 & 3.40 & I & 97.65 & 2.00 & I \\
\hline 38 & $24-02-21$ & 438.70 & 3.45 & I & 244.75 & -0.15 & D & 97.95 & 0.30 & I \\
\hline 39 & $25-02-21$ & 461.55 & 22.85 & I & 252.45 & 7.70 & I & 100.90 & 2.95 & I \\
\hline 40 & $26-02-21$ & 449.85 & -11.70 & $\mathrm{D}$ & 242.45 & -10.00 & $\mathrm{D}$ & 98.05 & -2.85 & $\mathrm{D}$ \\
\hline 41 & 01-03-21 & 455.25 & 5.40 & I & 247.5 & 5.05 & I & 100.70 & 2.65 & I \\
\hline 42 & $02-03-21$ & 469.00 & 13.75 & I & 250.1 & 2.60 & I & 102.20 & 1.50 & $\mathrm{I}$ \\
\hline 43 & $03-03-21$ & 466.35 & -2.65 & $\mathrm{D}$ & 248.9 & -1.20 & $\mathrm{D}$ & 102.60 & 0.40 & I \\
\hline 44 & 04-03-21 & 463.90 & -2.45 & $\mathrm{D}$ & 245.85 & -3.05 & $\mathrm{D}$ & 101.45 & -1.15 & $\mathrm{D}$ \\
\hline 45 & $05-03-21$ & 461.80 & -2.10 & $\mathrm{D}$ & 243.2 & -2.65 & $\mathrm{D}$ & 101.46 & 0.01 & I \\
\hline 46 & 08-03-21 & 466.70 & 4.90 & $\mathrm{I}$ & 243.15 & -0.05 & $\mathrm{D}$ & 103.60 & 2.14 & I \\
\hline 47 & 09-03-21 & 445.90 & -20.80 & $\mathrm{D}$ & 238.2 & -4.95 & $\mathrm{D}$ & 100.55 & -3.05 & $\mathrm{D}$ \\
\hline 48 & $10-03-21$ & 445.50 & -0.40 & $\mathrm{D}$ & 241 & 2.80 & $\mathrm{I}$ & 98.85 & -1.70 & $\mathrm{D}$ \\
\hline
\end{tabular}




\begin{tabular}{|c|c|c|c|c|c|c|c|c|c|c|}
\hline 49 & $12-03-21$ & 457.55 & 12.05 & I & 241.35 & 0.35 & I & 101.30 & 2.45 & I \\
\hline 50 & $15-03-21$ & 460.55 & 3.00 & I & 241.9 & 0.55 & I & 101.05 & -0.25 & $\mathrm{D}$ \\
\hline 51 & $16-03-21$ & 453.85 & -6.70 & $\mathrm{D}$ & 241.65 & -0.25 & $\mathrm{D}$ & 100.40 & -0.65 & $\mathrm{D}$ \\
\hline 52 & $17-03-21$ & 432.05 & -21.80 & $\mathrm{D}$ & 235.9 & -5.75 & $\mathrm{D}$ & 98.20 & -2.20 & $\mathrm{D}$ \\
\hline 53 & $18-03-21$ & 426.90 & -5.15 & $\mathrm{D}$ & 233.65 & -2.25 & $\mathrm{D}$ & 97.25 & -0.95 & $\mathrm{D}$ \\
\hline 54 & $19-03-21$ & 432.95 & 6.05 & I & 234.75 & 1.10 & I & 98.75 & 1.50 & $\mathrm{I}$ \\
\hline 55 & $22-03-21$ & 439.35 & 6.40 & I & 236.55 & 1.80 & I & 99.15 & 0.40 & I \\
\hline 56 & $23-03-21$ & 436.50 & -2.85 & $\mathrm{D}$ & 239 & 2.45 & I & 96.75 & -2.40 & $\mathrm{D}$ \\
\hline 57 & 24-03-21 & 429.95 & -6.55 & $\mathrm{D}$ & 232.5 & -6.50 & $\mathrm{D}$ & 94.55 & -2.20 & $\mathrm{D}$ \\
\hline 58 & $25-03-21$ & 418.95 & -11.00 & $\mathrm{D}$ & 227.25 & -5.25 & $\mathrm{D}$ & 90.90 & -3.65 & $\mathrm{D}$ \\
\hline 59 & $26-03-21$ & 424.20 & 5.25 & I & 229.8 & 2.55 & I & 90.10 & -0.80 & $\mathrm{D}$ \\
\hline 60 & $30-03-21$ & 430.80 & 6.60 & I & 230 & 0.20 & I & 91.55 & 1.45 & I \\
\hline 61 & $31-03-21$ & 427.95 & -2.85 & $\mathrm{D}$ & 234.5 & 4.50 & I & 91.85 & 0.30 & I \\
\hline 62 & 01-04-21 & 437.40 & 9.45 & I & 237.6 & 3.10 & I & 93.15 & 1.30 & I \\
\hline 63 & $05-04-21$ & 428.05 & -9.35 & $\mathrm{D}$ & 235.25 & -2.35 & $\mathrm{D}$ & 90.75 & -2.40 & $\mathrm{D}$ \\
\hline 64 & 06-04-21 & 428.10 & 0.05 & I & 235.4 & 0.15 & I & 91.15 & 0.40 & I \\
\hline 65 & 07-04-21 & 428.20 & 0.10 & I & 236.05 & 0.65 & I & 92.70 & 1.55 & I \\
\hline 66 & 08-04-21 & 430.55 & 2.35 & I & 238.75 & 2.70 & I & 92.95 & 0.25 & I \\
\hline 67 & 09-04-21 & 425.60 & -4.95 & $\mathrm{D}$ & 234.05 & -4.70 & $\mathrm{D}$ & 91.70 & -1.25 & $\mathrm{D}$ \\
\hline 68 & $12-04-21$ & 403.95 & -21.65 & $\mathrm{D}$ & 226.4 & -7.65 & $\mathrm{D}$ & 87.00 & -4.70 & $\mathrm{D}$ \\
\hline 69 & $13-04-21$ & 409.40 & 5.45 & I & 231.55 & 5.15 & I & 88.80 & 1.80 & I \\
\hline 70 & $15-04-21$ & 404.85 & -4.55 & $\mathrm{D}$ & 237.15 & 5.60 & I & 90.10 & 1.30 & I \\
\hline 71 & $16-04-21$ & 413.80 & 8.95 & I & 236.55 & -0.60 & $\mathrm{D}$ & 90.15 & 0.05 & I \\
\hline 72 & 19-04-21 & 408.75 & -5.05 & $\mathrm{D}$ & 231.4 & -5.15 & $\mathrm{D}$ & 88.10 & -2.05 & $\mathrm{D}$ \\
\hline 73 & $20-04-21$ & 408.10 & -0.65 & $\mathrm{D}$ & 232.7 & 1.30 & I & 87.80 & -0.30 & $\mathrm{D}$ \\
\hline 74 & $22-04-21$ & 418.35 & 10.25 & I & 233.5 & 0.80 & I & 87.85 & 0.05 & I \\
\hline 75 & $23-04-21$ & 423.05 & 4.70 & I & 233.7 & 0.20 & I & 88.05 & 0.20 & I \\
\hline 76 & $26-04-21$ & 418.90 & -4.15 & $\mathrm{D}$ & 232.5 & -1.20 & $\mathrm{D}$ & 88.20 & 0.15 & $\mathrm{I}$ \\
\hline 77 & 27-04-21 & 420.35 & 1.45 & $\mathrm{I}$ & 232.95 & 0.45 & I & 88.85 & 0.65 & $\mathrm{I}$ \\
\hline 78 & 28-04-21 & 417.50 & -2.85 & $\mathrm{D}$ & 231.35 & -1.60 & $\mathrm{D}$ & 89.40 & 0.55 & I \\
\hline 79 & 29-04-21 & 419.55 & 2.05 & I & 229.7 & -1.65 & D & 88.90 & -0.50 & D \\
\hline 80 & $30-04-21$ & 421.80 & 2.25 & I & 234.65 & 4.95 & I & 90.85 & 1.95 & I \\
\hline 81 & $03-05-21$ & 416.00 & -5.80 & $\mathrm{D}$ & 235.85 & 1.20 & I & 91.45 & 0.60 & $\mathrm{I}$ \\
\hline 82 & $04-05-21$ & 421.45 & 5.45 & I & 241.5 & 5.65 & I & 91.40 & -0.05 & $\mathrm{D}$ \\
\hline 83 & $05-05-21$ & 424.80 & 3.35 & I & 244.8 & 3.30 & I & 92.20 & 0.80 & $\mathrm{I}$ \\
\hline 84 & $06-05-21$ & 434.00 & 9.20 & $\mathrm{I}$ & 245.4 & 0.60 & I & 92.60 & 0.40 & I \\
\hline 85 & $07-05-21$ & 443.15 & 9.15 & I & 251.45 & 6.05 & I & 94.70 & 2.10 & I \\
\hline 86 & $10-05-21$ & 453.70 & 10.55 & I & 255 & 3.55 & I & 99.15 & 4.45 & I \\
\hline 87 & 11-05-21 & 462.25 & 8.55 & $\mathrm{I}$ & 265.6 & 10.60 & I & 103.70 & 4.55 & I \\
\hline 88 & $12-05-21$ & 449.25 & -13.00 & $\mathrm{D}$ & 262.25 & -3.35 & $\mathrm{D}$ & 102.80 & -0.90 & $\mathrm{D}$ \\
\hline 89 & $14-05-21$ & 442.25 & -7.00 & $\mathrm{D}$ & 254.4 & -7.85 & $\mathrm{D}$ & 101.05 & -1.75 & $\mathrm{D}$ \\
\hline
\end{tabular}




\begin{tabular}{|c|c|c|c|c|c|c|c|c|c|c|}
\hline 90 & $17-05-21$ & 443.65 & 1.40 & I & 251.85 & -2.55 & $\mathrm{D}$ & 102.00 & 0.95 & I \\
\hline 91 & $18-05-21$ & 448.45 & 4.80 & I & 265 & 13.15 & I & 105.55 & 3.55 & I \\
\hline 92 & $19-05-21$ & 446.45 & -2.00 & $\mathrm{D}$ & 272.75 & 7.75 & I & 107.20 & 1.65 & $\mathrm{I}$ \\
\hline 93 & $20-05-21$ & 454.80 & 8.35 & I & 266.4 & -6.35 & $\mathrm{D}$ & 104.70 & -2.50 & $\mathrm{D}$ \\
\hline 94 & $21-05-21$ & 461.05 & 6.25 & I & 276.3 & 9.90 & I & 104.30 & -0.40 & $\mathrm{D}$ \\
\hline 95 & $24-05-21$ & 472.85 & 11.80 & I & 284.55 & 8.25 & I & 109.45 & 5.15 & I \\
\hline 96 & $25-05-21$ & 474.40 & 1.55 & I & 288.15 & 3.60 & I & 110.30 & 0.85 & I \\
\hline 97 & $26-05-21$ & 472.20 & -2.20 & $\mathrm{D}$ & 284.25 & -3.90 & $\mathrm{D}$ & 112.00 & 1.70 & I \\
\hline 98 & $27-05-21$ & 467.85 & -4.35 & $\mathrm{D}$ & 280.4 & -3.85 & $\mathrm{D}$ & 110.25 & -1.75 & $\mathrm{D}$ \\
\hline 99 & $28-05-21$ & 471.50 & 3.65 & I & 280.65 & 0.25 & I & 109.85 & -0.40 & $\mathrm{D}$ \\
\hline 100 & $31-05-21$ & 472.00 & 0.50 & I & 279.7 & -0.95 & $\mathrm{D}$ & 109.25 & -0.60 & $\mathrm{D}$ \\
\hline 101 & 01-06-21 & 470.85 & -1.15 & $\mathrm{D}$ & 281.55 & 1.85 & I & 109.90 & 0.65 & I \\
\hline 102 & $02-06-21$ & 474.65 & 3.80 & I & 288.4 & 6.85 & I & 110.85 & 0.95 & I \\
\hline 103 & 03-06-21 & 473.90 & -0.75 & $\mathrm{D}$ & 287.65 & -0.75 & $\mathrm{D}$ & 112.45 & 1.60 & $\mathrm{I}$ \\
\hline 104 & 04-06-21 & 478.25 & 4.35 & I & 298.8 & 11.15 & I & 114.60 & 2.15 & I \\
\hline 105 & $07-06-21$ & 481.40 & 3.15 & I & 301.1 & 2.30 & I & 114.70 & 0.10 & I \\
\hline 106 & 08-06-21 & 487.45 & 6.05 & I & 302.25 & 1.15 & I & 116.70 & 2.00 & I \\
\hline 107 & 09-06-21 & 485.05 & -2.40 & $\mathrm{D}$ & 300.35 & -1.90 & $\mathrm{D}$ & 114.95 & -1.75 & $\mathrm{D}$ \\
\hline 108 & $10-06-21$ & 483.55 & -1.50 & $\mathrm{D}$ & 298.45 & -1.90 & $\mathrm{D}$ & 115.95 & 1.00 & $\mathrm{I}$ \\
\hline 109 & $11-06-21$ & 483.60 & 0.05 & I & 295.05 & -3.40 & $\mathrm{D}$ & 115.10 & -0.85 & $\mathrm{D}$ \\
\hline 110 & $14-06-21$ & 483.70 & 0.10 & I & 298.4 & 3.35 & I & 114.75 & -0.35 & $\mathrm{D}$ \\
\hline 111 & $15-06-21$ & 481.90 & -1.80 & $\mathrm{D}$ & 298.35 & -0.05 & $\mathrm{D}$ & 115.75 & 1.00 & I \\
\hline 112 & $16-06-21$ & 481.25 & -0.65 & $\mathrm{D}$ & 304.35 & 6.00 & I & 115.90 & 0.15 & I \\
\hline 113 & $17-06-21$ & 476.90 & -4.35 & $\mathrm{D}$ & 309 & 4.65 & I & 114.60 & -1.30 & $\mathrm{D}$ \\
\hline 114 & $18-06-21$ & 472.35 & -4.55 & $\mathrm{D}$ & 295.75 & -13.25 & $\mathrm{D}$ & 112.30 & -2.30 & $\mathrm{D}$ \\
\hline 115 & 21-06-21 & 475.05 & 2.70 & I & 302.4 & 6.65 & I & 112.65 & 0.35 & I \\
\hline 116 & $22-06-21$ & 473.65 & -1.40 & $\mathrm{D}$ & 304.55 & 2.15 & I & 113.85 & 1.20 & $\mathrm{I}$ \\
\hline 117 & $23-06-21$ & 469.60 & -4.05 & $\mathrm{D}$ & 300.25 & -4.30 & $\mathrm{D}$ & 112.90 & -0.95 & $\mathrm{D}$ \\
\hline 118 & $24-06-21$ & 466.10 & -3.50 & $\mathrm{D}$ & 295.4 & -4.85 & $\mathrm{D}$ & 111.45 & -1.45 & $\mathrm{D}$ \\
\hline 119 & $25-06-21$ & 470.05 & 3.95 & I & 298 & 2.60 & I & 111.40 & -0.05 & $\mathrm{D}$ \\
\hline 120 & $28-06-21$ & 467.15 & -2.90 & $\mathrm{D}$ & 297.25 & -0.75 & $\mathrm{D}$ & 110.85 & -0.55 & $\mathrm{D}$ \\
\hline 121 & 29-06-21 & 466.25 & -0.90 & $\mathrm{D}$ & 292.55 & -4.70 & $\mathrm{D}$ & 108.15 & -2.70 & $\mathrm{D}$ \\
\hline 122 & $30-06-21$ & 468.10 & 1.85 & $\mathrm{I}$ & 293.2 & 0.65 & I & 107.90 & -0.25 & $\mathrm{D}$ \\
\hline 123 & 01-07-21 & 462.75 & -5.35 & $\mathrm{D}$ & 296.2 & 3.00 & I & 107.55 & -0.35 & $\mathrm{D}$ \\
\hline 124 & $02-07-21$ & 462.76 & 0.01 & $\mathrm{I}$ & 297.95 & 1.75 & I & 108.45 & 0.90 & $\mathrm{I}$ \\
\hline 125 & 05-07-21 & 459.70 & -3.06 & $\mathrm{D}$ & 304.45 & 6.50 & $\mathrm{I}$ & 108.80 & 0.35 & I \\
\hline 126 & $06-07-21$ & 458.05 & -1.65 & $\mathrm{D}$ & 304.55 & 0.10 & I & 108.40 & -0.40 & $\mathrm{D}$ \\
\hline 127 & $07-07-21$ & 460.50 & 2.45 & I & 306.35 & 1.80 & I & 107.95 & -0.45 & $\mathrm{D}$ \\
\hline 128 & $08-07-21$ & 458.20 & -2.30 & $\mathrm{D}$ & 283.85 & -22.50 & $\mathrm{D}$ & 107.55 & -0.40 & $\mathrm{D}$ \\
\hline 129 & 09-07-21 & 456.90 & -1.30 & $\mathrm{D}$ & 278.95 & -4.90 & $\mathrm{D}$ & 107.30 & -0.25 & $\mathrm{D}$ \\
\hline 130 & $12-07-21$ & 451.00 & -5.90 & $\mathrm{D}$ & 271.9 & -7.05 & $\mathrm{D}$ & 107.20 & -0.10 & $\mathrm{D}$ \\
\hline
\end{tabular}




\begin{tabular}{|c|c|c|c|c|c|c|c|c|c|c|}
\hline 131 & $13-07-21$ & 450.25 & -0.75 & $\mathrm{D}$ & 272.2 & 0.30 & I & 107.80 & 0.60 & I \\
\hline 132 & $14-07-21$ & 448.70 & -1.55 & $\mathrm{D}$ & 270.1 & -2.10 & $\mathrm{D}$ & 107.10 & -0.70 & $\mathrm{D}$ \\
\hline 133 & $15-07-21$ & 447.05 & -1.65 & $\mathrm{D}$ & 267.1 & -3.00 & $\mathrm{D}$ & 106.30 & -0.80 & $\mathrm{D}$ \\
\hline 134 & $16-07-21$ & 448.00 & 0.95 & I & 268.1 & 1.00 & I & 106.10 & -0.20 & $\mathrm{D}$ \\
\hline 135 & $19-07-21$ & 453.90 & 5.90 & I & 272.05 & 3.95 & I & 105.95 & -0.15 & $\mathrm{D}$ \\
\hline 136 & $20-07-21$ & 453.80 & -0.10 & $\mathrm{D}$ & 276.15 & 4.10 & $\mathrm{I}$ & 105.60 & -0.35 & $\mathrm{D}$ \\
\hline 137 & $22-07-21$ & 460.90 & 7.10 & I & 276.4 & 0.25 & I & 106.70 & 1.10 & I \\
\hline 138 & $23-07-21$ & 461.35 & 0.45 & I & 277.4 & 1.00 & I & 106.00 & -0.70 & $\mathrm{D}$ \\
\hline 139 & $26-07-21$ & 456.00 & -5.35 & $\mathrm{D}$ & 273.55 & -3.85 & $\mathrm{D}$ & 104.65 & -1.35 & $\mathrm{D}$ \\
\hline 140 & $27-07-21$ & 453.30 & -2.70 & $\mathrm{D}$ & 269.2 & -4.35 & $\mathrm{D}$ & 103.95 & -0.70 & $\mathrm{D}$ \\
\hline 141 & $28-07-21$ & 453.45 & 0.15 & I & 264.95 & -4.25 & $\mathrm{D}$ & 104.00 & 0.05 & I \\
\hline 142 & $29-07-21$ & 449.10 & -4.35 & $\mathrm{D}$ & 263.6 & -1.35 & $\mathrm{D}$ & 103.90 & -0.10 & $\mathrm{D}$ \\
\hline 143 & $30-07-21$ & 445.50 & -3.60 & $\mathrm{D}$ & 261.15 & -2.45 & $\mathrm{D}$ & 103.15 & -0.75 & $\mathrm{D}$ \\
\hline 144 & $02-08-21$ & 457.80 & 12.30 & I & 272.8 & 11.65 & I & 105.50 & 2.35 & I \\
\hline 145 & 03-08-21 & 463.40 & 5.60 & I & 274.65 & 1.85 & I & 106.15 & 0.65 & I \\
\hline 146 & 04-08-21 & 457.15 & -6.25 & $\mathrm{D}$ & 272.25 & -2.40 & $\mathrm{D}$ & 105.30 & -0.85 & $\mathrm{D}$ \\
\hline 147 & $05-08-21$ & 453.50 & -3.65 & $\mathrm{D}$ & 265 & -7.25 & $\mathrm{D}$ & 103.95 & -1.35 & $\mathrm{D}$ \\
\hline 148 & 06-08-21 & 455.60 & 2.10 & I & 264.95 & -0.05 & $\mathrm{D}$ & 105.90 & 1.95 & I \\
\hline 149 & 09-08-21 & 452.50 & -3.10 & $\mathrm{D}$ & 262 & -2.95 & $\mathrm{D}$ & 104.40 & -1.50 & $\mathrm{D}$ \\
\hline 150 & $10-08-21$ & 444.70 & -7.80 & $\mathrm{D}$ & 257.95 & -4.05 & $\mathrm{D}$ & 102.25 & -2.15 & $\mathrm{D}$ \\
\hline 151 & 11-08-21 & 450.00 & 5.30 & $\mathrm{I}$ & 258.9 & 0.95 & I & 104.65 & 2.40 & I \\
\hline 152 & $12-08-21$ & 448.15 & -1.85 & $\mathrm{D}$ & 255.2 & -3.70 & $\mathrm{D}$ & 104.10 & -0.55 & $\mathrm{D}$ \\
\hline 153 & $13-08-21$ & 454.90 & 6.75 & I & 255.05 & -0.15 & $\mathrm{D}$ & 104.65 & 0.55 & I \\
\hline 154 & $16-08-21$ & 464.00 & 9.10 & I & 257.7 & 2.65 & I & 107.20 & 2.55 & I \\
\hline 155 & $17-08-21$ & 465.00 & 1.00 & $\mathrm{I}$ & 253.6 & -4.10 & $\mathrm{D}$ & 105.70 & -1.50 & $\mathrm{D}$ \\
\hline 156 & $18-08-21$ & 463.50 & -1.50 & $\mathrm{D}$ & 253.8 & 0.20 & I & 106.20 & 0.50 & I \\
\hline 157 & $20-08-21$ & 455.20 & -8.30 & $\mathrm{D}$ & 247.2 & -6.60 & $\mathrm{D}$ & 103.50 & -2.70 & $\mathrm{D}$ \\
\hline 158 & $23-08-21$ & 451.70 & -3.50 & $\mathrm{D}$ & 245.65 & -1.55 & $\mathrm{D}$ & 103.00 & -0.50 & $\mathrm{D}$ \\
\hline 159 & $24-08-21$ & 456.40 & 4.70 & $\mathrm{I}$ & 249.75 & 4.10 & I & 105.90 & 2.90 & $\mathrm{I}$ \\
\hline 160 & $25-08-21$ & 460.70 & 4.30 & I & 257.85 & 8.10 & I & 106.10 & 0.20 & I \\
\hline 161 & $26-08-21$ & 466.75 & 6.05 & $\mathrm{I}$ & 255.5 & -2.35 & D & 105.60 & -0.50 & $\mathrm{D}$ \\
\hline 162 & $27-08-21$ & 471.30 & 4.55 & I & 256.6 & 1.10 & I & 105.95 & 0.35 & I \\
\hline 163 & $30-08-21$ & 472.55 & 1.25 & I & 259.6 & 3.00 & I & 107.35 & 1.40 & $\mathrm{I}$ \\
\hline 164 & $31-08-21$ & 471.65 & -0.90 & $\mathrm{D}$ & 266.6 & 7.00 & I & 110.85 & 3.50 & I \\
\hline 165 & 01-09-21 & 477.00 & 5.35 & I & 266.65 & 0.05 & I & 110.75 & -0.10 & $\mathrm{D}$ \\
\hline 166 & $02-09-21$ & 479.80 & 2.80 & I & 266.7 & 0.05 & I & 110.15 & -0.60 & $\mathrm{D}$ \\
\hline 167 & 03-09-21 & 491.30 & 11.50 & $\mathrm{I}$ & 275.95 & 9.25 & $\mathrm{I}$ & 113.10 & 2.95 & $\mathrm{I}$ \\
\hline 168 & 06-09-21 & 490.80 & -0.50 & $\mathrm{D}$ & 269.95 & -6.00 & $\mathrm{D}$ & 111.60 & -1.50 & $\mathrm{D}$ \\
\hline 169 & 07-09-21 & 481.95 & -8.85 & $\mathrm{D}$ & 267.35 & -2.60 & $\mathrm{D}$ & 111.85 & 0.25 & I \\
\hline 170 & 08-09-21 & 489.55 & 7.60 & I & 269.85 & 2.50 & I & 112.60 & 0.75 & I \\
\hline 171 & 09-09-21 & 491.15 & 1.60 & $\mathrm{I}$ & 268.65 & -1.20 & $\mathrm{D}$ & 112.80 & 0.20 & $\mathrm{I}$ \\
\hline
\end{tabular}




\begin{tabular}{|c|c|c|c|c|c|c|c|c|c|c|}
\hline 172 & $13-09-21$ & 498.10 & 6.95 & $\mathrm{I}$ & 270.65 & 2.00 & $\mathrm{I}$ & 114.00 & 1.20 & $\mathrm{I}$ \\
\hline 173 & $14-09-21$ & 493.10 & -5.00 & $\mathrm{D}$ & 274.15 & 3.50 & $\mathrm{I}$ & 115.25 & 1.25 & $\mathrm{I}$ \\
\hline 174 & $15-09-21$ & 489.95 & -3.15 & $\mathrm{D}$ & 275.7 & 1.55 & $\mathrm{I}$ & 116.40 & 1.15 & $\mathrm{I}$ \\
\hline 175 & $16-09-21$ & 439.05 & -50.90 & $\mathrm{D}$ & 282.5 & 6.80 & $\mathrm{I}$ & 118.35 & 1.95 & $\mathrm{I}$ \\
\hline 176 & $17-09-21$ & 436.25 & -2.80 & $\mathrm{D}$ & 282.95 & 0.45 & $\mathrm{I}$ & 118.25 & -0.10 & $\mathrm{D}$ \\
\hline 177 & $20-09-21$ & 420.40 & -15.85 & $\mathrm{D}$ & 272.50 & -10.45 & $\mathrm{D}$ & 114.60 & -3.65 & $\mathrm{D}$ \\
\hline
\end{tabular}

\section{BPCL.NS}

Transition Frequency Matrix of BPCL.NS

$$
F=\left[\begin{array}{ll}
45 & 43 \\
43 & 44
\end{array}\right] \text {, and }
$$

Transition probability matrix is

$P=\left[\begin{array}{ll}45 / 88 & 43 / 88 \\ 43 / 87 & 44 / 87\end{array}\right]$

$P=\left[\begin{array}{ll}0.51136 & 0.48863 \\ 0.49425 & 0.50574\end{array}\right]$,

$P^{2}=\left[\begin{array}{cc}0.503003 & 0.496997 \\ 0.50271 & 0.49729\end{array}\right]$,

$P^{3}=\left[\begin{array}{cc}0.50286 & 0.49714 \\ 0.502855 & 0.497145\end{array}\right]$,

$P^{4}=\left[\begin{array}{ll}0.502857 & 0.497143 \\ 0.502857 & 0.497143\end{array}\right]$,

$P^{n}=\left[\begin{array}{ll}0.502857 & 0.497143 \\ 0.502857 & 0.497143\end{array}\right]$

\section{HINDPETRO.NS}

Transition Frequency Matrix of HINDPETRO.NS

$$
F=\left[\begin{array}{ll}
57 & 40 \\
39 & 39
\end{array}\right], \text { and }
$$

Transition probability matrix is

$$
\begin{gathered}
P=\left[\begin{array}{ll}
57 / 97 & 40 / 97 \\
39 / 78 & 39 / 78
\end{array}\right] \\
P=\left[\begin{array}{cc}
0.587629 & 0.412371 \\
0.5 & 0.5
\end{array}\right], \\
P^{2}=\left[\begin{array}{ll}
0.551493 & 0.448507 \\
0.543814 & 0.456186
\end{array}\right], \\
P^{3}=\left[\begin{array}{ll}
0.548327 & 0.451673 \\
0.547654 & 0.452346
\end{array}\right], \\
P^{4}=\left[\begin{array}{cc}
0.548049 & 0.451951 \\
0.54799 & 0.45201
\end{array}\right], \\
P^{5}=\left[\begin{array}{cc}
0.548025 & 0.451975 \\
0.54802 & 0.45198
\end{array}\right], \\
P^{6}=\left[\begin{array}{cc}
0.548023 & 0.451977 \\
0.548022 & 0.451978
\end{array}\right], \\
P^{7}=\left[\begin{array}{cc}
0.548023 & 0.451977 \\
0.54802 & 0.451977
\end{array}\right], \\
\cdot
\end{gathered}
$$

\section{IOC.NS}

Transition Frequency Matrix of

IOC.NS is

$$
F=\left[\begin{array}{ll}
54 & 36 \\
37 & 48
\end{array}\right], \text { and }
$$

Transition probability matrix is

$$
\begin{array}{r}
P=\left[\begin{array}{ll}
54 / 90 & 36 / 90 \\
37 / 85 & 48 / 85
\end{array}\right] \\
P=\left[\begin{array}{cc}
0.6 & 0.4 \\
0.435294 & 0.564706
\end{array}\right], \\
P^{2}=\left[\begin{array}{cc}
0.534118 & 0.465882 \\
0.50699 & 0.49301
\end{array}\right], \\
P^{3}=\left[\begin{array}{ll}
0.523266 & 0.476734 \\
0.518798 & 0.481202
\end{array}\right], \\
P^{4}=\left[\begin{array}{ll}
0.521479 & 0.478521 \\
0.520743 & 0.479257
\end{array}\right], \\
P^{5}=\left[\begin{array}{ll}
0.521185 & 0.478815 \\
0.521064 & 0.478936
\end{array}\right], \\
P^{6}=\left[\begin{array}{ll}
0.521136 & 0.478864 \\
0.521116 & 0.478884
\end{array}\right], \\
P^{7}=\left[\begin{array}{ll}
0.521128 & 0.478872 \\
0.521126 & 0.478874
\end{array}\right], \\
P^{8}=\left[\begin{array}{ll}
0.521127 & 0.478873 \\
0.521126 & 0.478874
\end{array}\right], \\
P^{9}=\left[\begin{array}{ll}
0.521127 & 0.478873 \\
0.521127 & 0.478873
\end{array}\right], \\
\cdot
\end{array}
$$

From the $P^{2}$ to $P^{n}$ values, we observed that the probability of decrease to decrease, decrease to increase, increase to decrease and increase to increase values having same probability in $4^{\text {th }}$ subsequence days in BPCL.NS, the next stock close value such as same probability in $7^{\text {th }}$ subsequence days in HINDPETRO.NS and another one stock close value same probability in $9^{\text {th }}$ subsequence days in IOC.NS. From this empirical evidence, Markov chain is more useful and powerful to predict next day values; it is not reliable for subsequent days. 
In my observation all the above mentioned three types of Stochastic models were partially supported to prediction of the stock market, even some of the authors were strongly agree with the stock market prediction is not reliable to the investors. Some of the authors said that, their prediction method was more reliable for short term investors only. The above empirical result also shows that the Markov chain analysis is powerful tool for predict next day value only, which is not useful for lateral days.

In this paper, BPCL.NS, HINDPETRO.NS and IOC.NS daily close value data for a month period is considered. Two observing symbols " $\mathrm{I}$ " and " $\mathrm{D}$ " have been used.

where, "I indicates Increasing”, “ D indicates Decreasing”

If Today's close value - Yesterday's close value $>0$, then observing symbol is I

If Today's close value - Yesterday's close value $<0$ then observing symbol is D

Minimum value is: -63.40 , Maximum values is: $145.48, \mathrm{D}_{1}: 3$ and $\mathrm{D}_{2}: 4.55$

$$
\mathrm{U}=\left[\mathrm{D}_{\min }-\mathrm{D}_{1}, \mathrm{D}_{\max }+\mathrm{D}_{2}\right]
$$

where, $D_{\min }$ is Minimum value, $D_{\max }$ is Maximum value, $D_{1}$ and $D_{2}$ is two positive real number.

$$
\left.\left.\mathrm{U}:[-160 \text { to }-150], \mathrm{U}_{1} \text { : [-160 to }-15\right], \mathrm{U}_{2} \text { : [-15 to 0], } \mathrm{U}_{3} \text { : [0 to } 20 \text { ] and } \mathrm{U}_{4} \text { : [20 to } 150\right] \text {. }
$$

There are four hidden states assumed and are denoted by the symbol as S1, S2, S3, S4. where, S1 - very low, S2 - low, S3 - high and S4 - very high

\section{$\underline{B P C L . N S}$}

Table 3: Transition table with probability values for difference in one day close value.

\begin{tabular}{ccccccccc} 
& S1 & \multicolumn{3}{c}{ S2 } & \multicolumn{3}{c}{ S3 } & S4 \\
\hline & I & D & I & D & I & D & I & D \\
\hline S1 & 0 & 0 & 0 & 0.7500 & 0.2500 & 0 & 0 & 0 \\
\hline S2 & 0 & 0.0366 & 0 & 0.4512 & 0.5000 & 0 & 0.0122 & 0 \\
\hline S3 & 0 & 0.01 & 0 & 0.4713 & 0.5057 & 0 & 0.0115 & 0 \\
\hline S4 & 0 & 0 & 0 & 1.00 & 0 & 0 & 0 & 0 \\
\hline
\end{tabular}

Probability values of TPM, EPM, and $\pi$ for difference in day one close value (Figure 2 and Table 3):

$\left[\begin{array}{ccccc} & S 1 & S 2 & S 3 & S 4 \\ S 1 & 0 & 0.75 & 0.25 & 0 \\ S 2 & 0.04 & 0.45 & 0.50 & 0.01 \\ S 3 & 0.01 & 0.47 & 0.51 & 0.01 \\ S 4 & 0 & 1 & 0 & 0\end{array}\right] \quad$ and $\quad\left[\begin{array}{ccc} & I & D \\ S 1 & 0 & 1 \\ S 2 & 0.53846 & 0.46154 \\ S 3 & 0.375 & 0.625 \\ S 4 & 0 & 1\end{array}\right]$

Steady state probability distribution difference in day one close value.

$\pi=\left[\begin{array}{llll}0.0229 & 0.4742 & 0.4913 & 0.0114\end{array}\right]$ 


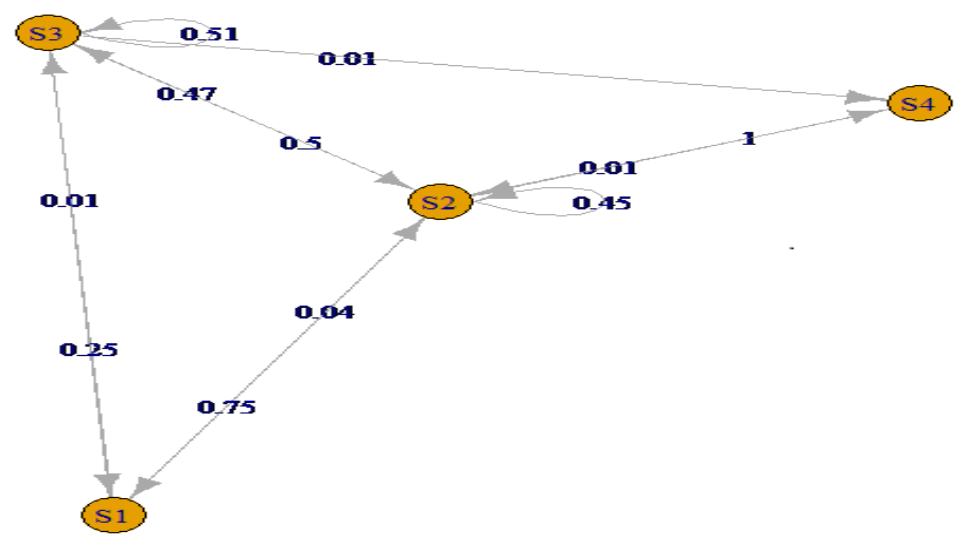

Figure 2. TPM of day one in BPCL.NS.

Table 4: Transition table with probability values for difference in day two close value.

\begin{tabular}{ccccccccc} 
& S1 & \multicolumn{3}{c}{ S2 } & \multicolumn{3}{c}{ S3 } & I \\
\hline & I & D & I & D & I & D & D \\
\hline S1 & 0 & 0 & 0 & 0.181818 & 0.545455 & 0 & 0.272727 & 0 \\
\hline S2 & 0 & 0.05 & 0 & 0.35 & 0.57333 & 0 & 0.03 & 0 \\
\hline S3 & 0 & 0.060241 & 0 & 0.542169 & 0.39759 & 0 & 0 & 0 \\
\hline S4 & 0 & 0.20 & 0 & 0.60 & 0.2 & 0 & 0 & 0 \\
\hline
\end{tabular}

Probability values of TPM, EPM, and $\pi$ for difference in day two close value (Figure 3 and Table 4):

$\left[\begin{array}{ccccc} & S 1 & S 2 & S 3 & S 4 \\ S 1 & 0 & 0.181818 & 0.545455 & 0.272727 \\ S 2 & 0.05 & 0.35 & 0.57333 & 0.03 \\ S 3 & 0.060241 & 0.542169 & 0.39759 & 0 \\ S 4 & 0.20 & 0.60 & 0.2 & 0\end{array}\right]$ and $\quad\left[\begin{array}{ccc} & I & D \\ S 1 & 0.818182 & 0.181818 \\ S 2 & 0.60 & 0.40 \\ S 3 & 0.39759 & 0.60241 \\ S 4 & 0.20 & 0.8\end{array}\right]$

Steady state probability distribution difference in day two close value.

$\pi=\left[\begin{array}{llll}0.0575 & 0.4374 & 0.4775 & 0.0273\end{array}\right]$

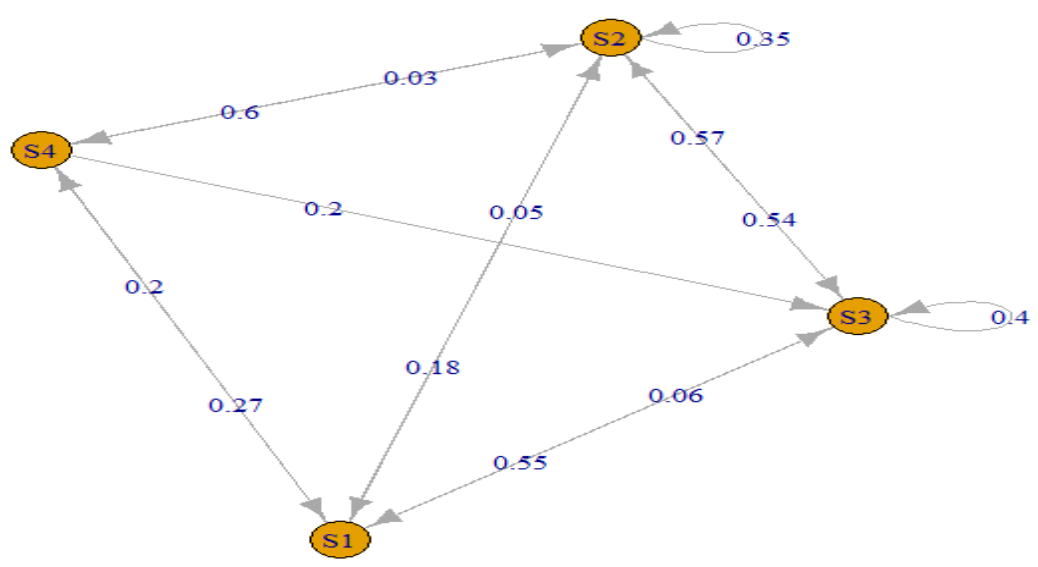

Figure 3. TPM of day two in BPCL.NS. 
Table 5: Transition table with probability values for difference in day three close value.

\begin{tabular}{ccccccccc} 
& S1 & \multicolumn{3}{c}{ S2 } & \multicolumn{3}{c}{ S3 } & \multicolumn{2}{c}{ S4 } \\
\hline & I & D & I & D & I & D & I & D \\
\hline S1 & 0 & 0 & 0 & 0.15 & 0.45 & 0 & 0.4 & 0 \\
\hline S2 & 0 & 0.06 & 0 & 0.238095 & 0.619048 & 0 & 0.079365 & 0 \\
\hline S3 & 0 & 0.12 & 0 & 0.533333 & 0.32 & 0 & 0.026667 & 0 \\
\hline S4 & 0 & 0.53 & 0 & 0.333333 & 0.133333 & 0 & 0 & 0 \\
\hline
\end{tabular}

Probability values of TPM, EPM, and $\boldsymbol{\pi}$ for difference in day three close value (Figure 4 and Table 5):

$\left[\begin{array}{ccccc} & S 1 & S 2 & S 3 & S 4 \\ S 1 & 0 & 0.15 & 0.45 & 0.4 \\ S 2 & 0.06 & 0.238095 & 0.619048 & 0.079365 \\ S 3 & 0.12 & 0.533333 & 0.32 & 0.026667 \\ S 4 & 0.53 & 0.333333 & 0.133333 & 0\end{array}\right] \quad$ and $\quad\left[\begin{array}{ccc} & I & D \\ S 1 & 0.85 & 0.15 \\ S 2 & 0.698413 & 0.30 \\ S 3 & 0.346667 & 0.653333 \\ S 4 & 0.133333 & 0.866667\end{array}\right]$

Steady state probability distribution difference in day Three close value.

$\pi=\left[\begin{array}{llll}0.1216 & 0.3620 & 0.4275 & 0.0887\end{array}\right]$

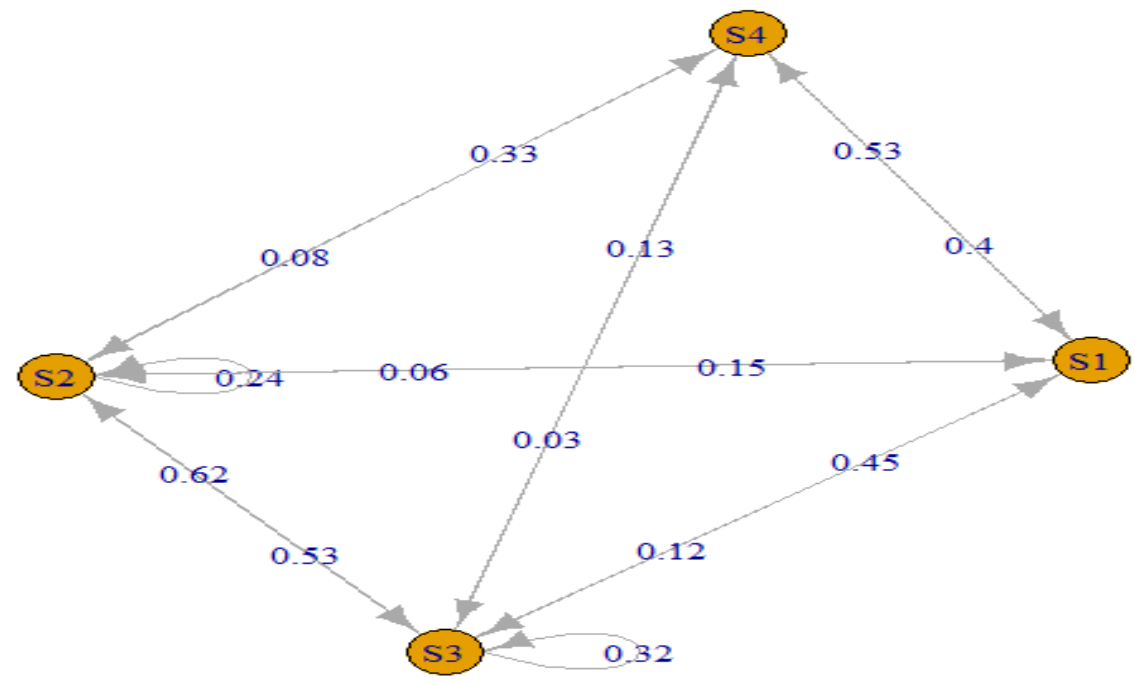

Figure 4. TPM of day three in BPCL.NS.

Table 6: Transition table with probability values for difference in day four close value.

\begin{tabular}{ccccccccc} 
& S1 & & S2 & & S3 & S4 & \\
\hline & I & D & I & D & I & D & I & D \\
\hline S1 & 0 & 0.000000 & 0 & 0.15 & 0.45 & 0 & 0.4 & 0 \\
\hline S2 & 0 & 0.063492 & 0 & 0.238095 & 0.619048 & 0 & 0.079365 & 0 \\
\hline S3 & 0 & 0.12 & 0 & 0.533333 & 0.32 & 0 & 0.026667 & 0 \\
\hline S4 & 0 & 0.533333 & 0 & 0.333333 & 0.133333 & 0 & 0 & 0 \\
\hline
\end{tabular}


Probability values of TPM, EPM, and $\pi$ for difference in day four close value (Figure 5 and Table 6):

$\left[\begin{array}{ccccc} & S 1 & S 2 & S 3 & S 4 \\ S 1 & 0 & 0.15 & 0.45 & 0.4 \\ S 2 & 0.063492 & 0.238095 & 0.619048 & 0.079365 \\ S 3 & 0.12 & 0.533333 & 0.32 & 0.026667 \\ S 4 & 0.533333 & 0.333333 & 0.133333 & 0\end{array}\right]$ and $\left[\begin{array}{ccc} & I & D \\ S 1 & 0.85 & 0.15 \\ S 2 & 0.698413 & 0.30 \\ S 3 & 0.346667 & 0.653333 \\ S 4 & 0.133333 & 0.866667\end{array}\right]$

Steady state probability distribution difference in day Four close value.

$\pi=\left[\begin{array}{llll}0.1216 & 0.3620 & 0.4275 & 0.0887\end{array}\right]$

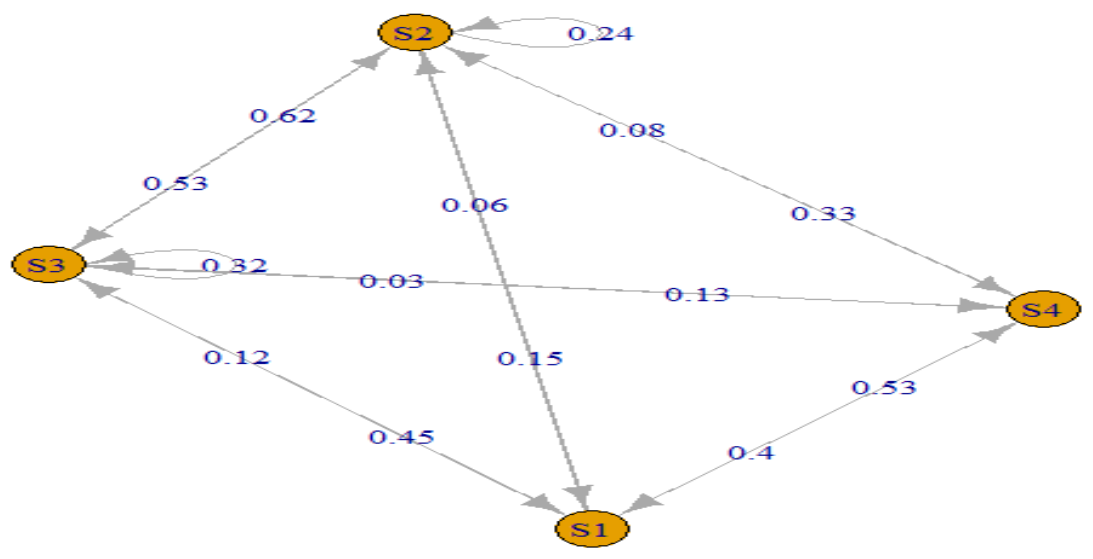

Figure 5. TPM of day four in BPCL.NS.

\section{HINDPETRO.NS}

Table 7: Transition table with probability values for difference in day one close value.

\begin{tabular}{ccccccccc} 
& S1 & & S2 & & S3 & \multicolumn{2}{c}{ S4 } \\
\hline & I & D & I & D & I & D & I & D \\
\hline S1 & 0 & 0 & 0 & 1 & 0 & 0 & 0 & 0 \\
\hline S2 & 0 & 0 & 0 & 0.506329 & 0.481013 & 0 & 0.012658 & 0 \\
\hline S3 & 0 & 0.01087 & 0 & 0.413044 & 0.576087 & 0 & 0 & 0 \\
\hline S4 & 0 & 0 & 0 & 0 & 1 & 0 & 0 & 0 \\
\hline
\end{tabular}

Probability values of TPM, EPM, and $\pi$ for difference in day one close value (Figure 6 and Table 7):

$\left[\begin{array}{ccccc} & S 1 & S 2 & S 3 & S 4 \\ S 1 & 0 & 1 & 0 & 0 \\ S 2 & 0 & 0.5063 & 0.481013 & 0.012658 \\ S 3 & 0.01087 & 0.41304 & 0.576087 & 0 \\ S 4 & 0 & 0 & 1 & 0\end{array}\right] \quad$ and $\quad\left[\begin{array}{ccc} & I & D \\ S 1 & 0 & 1 \\ S 2 & 0.493671 & 0.5063 \\ S 3 & 0.576087 & 0.4239 \\ S 4 & 1 & 0\end{array}\right]$


Steady state probability distribution difference in day one close value.

$\pi=\left[\begin{array}{llll}0.0057 & 0.4566 & 0.5317 & 0.0057\end{array}\right]$

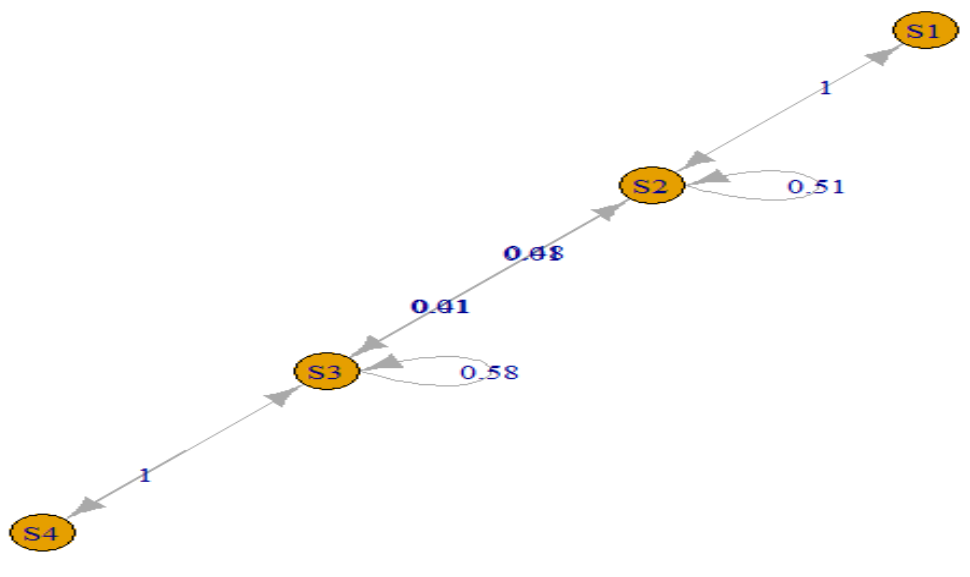

Figure 6. TPM of day one in HINDPETRO.NS

Table 8: Transition table with probability values for difference in day two close value.

\begin{tabular}{ccccccccc} 
& S1 & & S2 & & S3 & S4 & \\
\hline & I & D & I & D & I & D & I & D \\
\hline S1 & 0 & 0 & 0 & 0.2 & 0.8 & 0 & 0 & 0 \\
\hline S2 & 0 & 0.0120 & 0 & 0.373494 & 0.614458 & 0 & 0 & 0 \\
\hline S3 & 0 & 0.035294 & 0 & 0.60 & 0.352941 & 0 & 0.011765 & 0 \\
\hline S4 & 0 & 1 & 0 & 0 & 0 & 0 & 0 & 0 \\
\hline
\end{tabular}

Probability values of TPM, EPM, and $\pi$ for difference in day two close value (Figure 7 and Table 8):

$\left[\begin{array}{ccccc} & S 1 & S 2 & S 3 & S 4 \\ S 1 & 0 & 0.2 & 0.8 & 0 \\ S 2 & 0.0120 & 0.373494 & 0.614458 & 0 \\ S 3 & 0.03529 & 0.60 & 0.352941 & 0.011765 \\ S 4 & 1 & 0 & 0 & 0\end{array}\right]$ and $\left[\begin{array}{ccc} & I & D \\ S 1 & 0.80 & 0.20 \\ S 2 & 0.614458 & 0.3855 \\ S 3 & 0.364706 & 0.64 \\ S 4 & 0 & 1\end{array}\right]$

Steady state probability distribution difference in day two close value.

$\pi=\left[\begin{array}{llll}0.0287 & 0.4770 & 0.4885 & 0.0057\end{array}\right]$ 


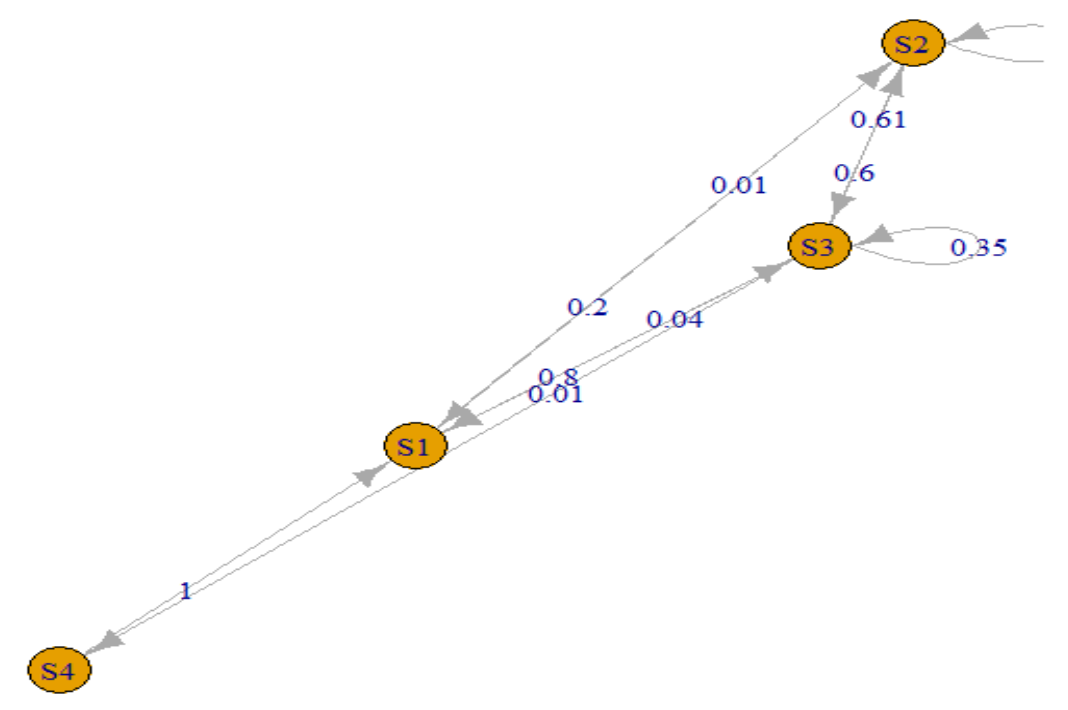

Figure 7. TPM of day two in HINDPETRO.NS

Table 9: Transition table with probability values for difference in day three close value.

\begin{tabular}{ccccccccc} 
& S1 & & S2 & & S3 & \multicolumn{2}{c}{ S4 } \\
\hline & I & D & I & D & I & D & I & D \\
\hline S1 & 0 & 0 & 0 & 0.25 & 0.5 & 0 & 0.25 & 0 \\
\hline S2 & 0 & 0.0290 & 0 & 0.289855 & 0.681159 & 0 & 0 & 0 \\
\hline S3 & 0 & 0.109756 & 0 & 0.548781 & 0.329268 & 0 & 0.012195 & 0 \\
\hline S4 & 0 & 0.83333 & 0 & 0 & 0 & 0 & 0.166667 & 0 \\
\hline
\end{tabular}

Probability values of TPM, EPM, and $\pi$ for difference in day three close value (Figure 8 and Table 9):

$\left[\begin{array}{ccccc} & S 1 & S 2 & S 3 & S 4 \\ S 1 & 0 & 0.25 & 0.5 & 0.25 \\ S 2 & 0.0290 & 0.289855 & 0.681159 & 0 \\ S 3 & 0.109756 & 0.548781 & 0.329268 & 0.012195 \\ S 4 & 0.83333 & 0 & 0 & 0.166667\end{array}\right]$ and $\left[\begin{array}{ccc} & I & D \\ S 1 & 0.75 & 0.25 \\ S 2 & 0.681159 & 0.3188 \\ S 3 & 0.341463 & 0.658537 \\ S 4 & 0.166667 & 0.833333\end{array}\right]$

Steady state probability distribution difference in day three close value.

$\pi=\left[\begin{array}{llll}0.0924 & 0.3988 & 0.4739 & 0.0346\end{array}\right]$ 


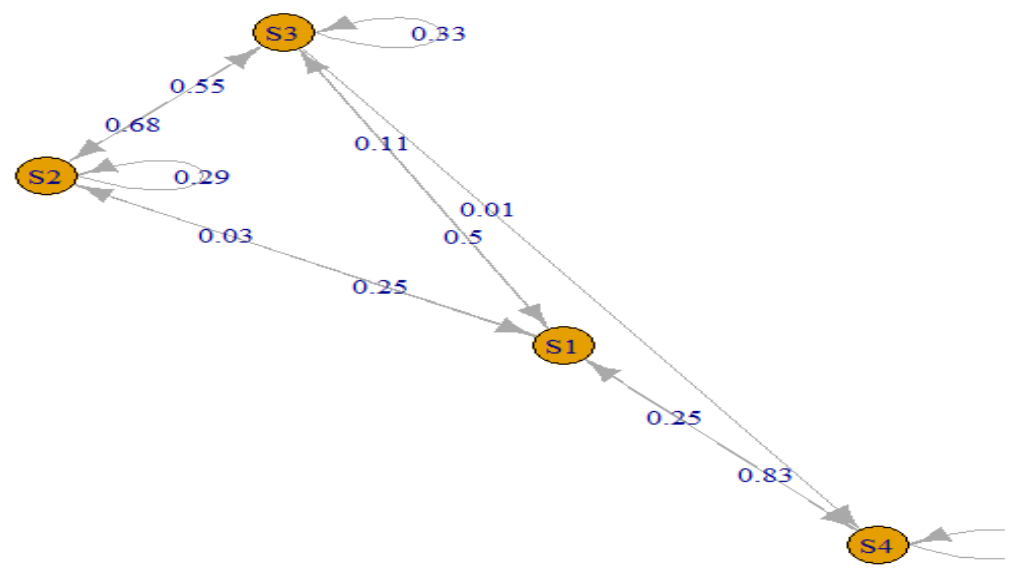

Figure 8. TPM of day three in HINDPETRO.NS

Table 10: Transition table with probability values for difference in day four close value.

\begin{tabular}{ccccccccc} 
& S1 & \multicolumn{3}{c}{ S2 } & \multicolumn{3}{c}{ S3 } & S4 \\
\hline & I & D & I & D & I & D & I & D \\
\hline S1 & 0 & 0.00 & 0 & 0.085714 & 0.514286 & 0 & 0.40 & 0 \\
\hline S2 & 0 & 0.06 & 0 & 0.351852 & 0.462963 & 0 & 0.12963 & 0 \\
\hline S3 & 0 & 0.322581 & 0 & 0.403226 & 0.274194 & 0 & 0 & 0 \\
\hline S4 & 0 & 0.571429 & 0 & 0.333333 & 0.095238 & 0 & 0 & 0 \\
\hline
\end{tabular}

Probability values of TPM, EPM, and $\pi$ for difference in day four close value (Figure 9 and Table 10):

$\left[\begin{array}{ccccc} & S 1 & S 2 & S 3 & S 4 \\ S 1 & 0 & 0.085714 & 0.514286 & 0.4 \\ S 2 & 0.06 & 0.351852 & 0.462963 & 0.12963 \\ S 3 & 0.322581 & 0.403226 & 0.274194 & 0 \\ S 4 & 0.571429 & 0.333333 & 0.095238 & 0\end{array}\right]$ and $\left[\begin{array}{ccc} & I & D \\ S 1 & 0.914286 & 0.085714 \\ S 2 & 0.592593 & 0.41 \\ S 3 & 0.274194 & 0.725806 \\ S 4 & 0.095238 & 0.904762\end{array}\right]$

Steady state probability distribution difference in day four close value.

$\pi=\left[\begin{array}{llll}0.2034 & 0.3139 & 0.3604 & 0.1220\end{array}\right]$

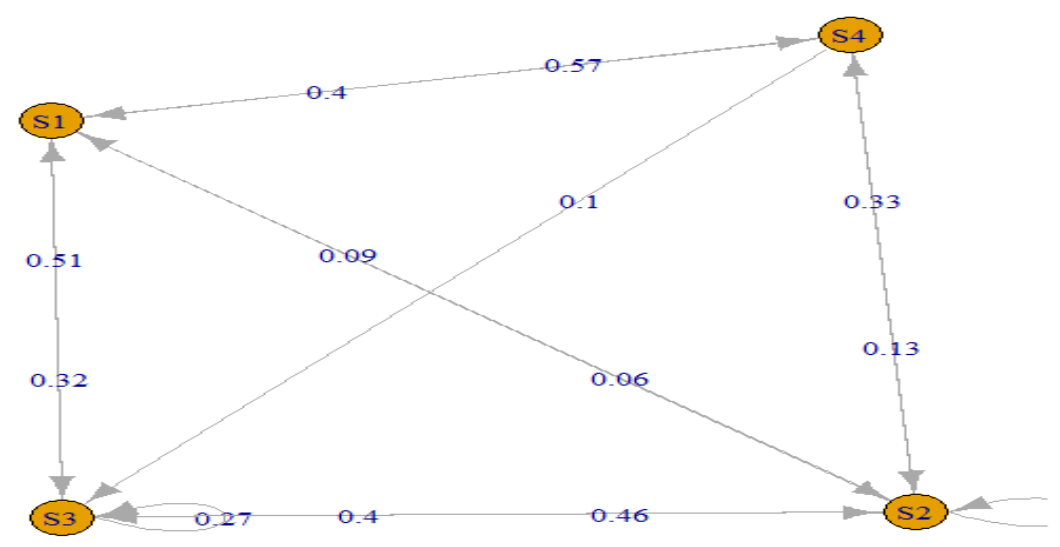

Figure 9. TPM of day four in HINDPETRO.NS 
IOC.NS

Table 11: Transition table with probability values for difference in day one close value.

\begin{tabular}{ccccccccc} 
& S1 & & S2 & \multicolumn{3}{c}{ S3 } & S4 & \\
\hline & I & D & I & D & I & D & I & D \\
\hline S1 & 0 & 0 & 0 & 0 & 0 & 0 & 1 & 0 \\
\hline S2 & 0 & 0 & 0 & 0.54878 & 0.45122 & 0 & 0 & 0 \\
\hline S3 & 0 & 0.01099 & 0 & 0.395604 & 0.593407 & 0 & 0 & 0 \\
\hline S4 & 0 & 0 & 0 & 1 & 0 & 0 & 0 & 0 \\
\hline
\end{tabular}

Probability values of TPM, EPM, and $\boldsymbol{\pi}$ for difference in day one close value (Figure 10 and Table 11):

$\left[\begin{array}{ccccc} & S 1 & S 2 & S 3 & S 4 \\ S 1 & 0 & 0 & 0 & 1 \\ S 2 & 0 & 0.54878 & 0.45122 & 0 \\ S 3 & 0.01099 & 0.395604 & 0.593407 & 0 \\ S 4 & 0 & 1 & 0 & 0\end{array}\right]$ and $\left[\begin{array}{ccc} & I & D \\ S 1 & 1 & 0 \\ S 2 & 0.456122 & 0.54878 \\ S 3 & 0.593407 & 0.40659 \\ S 4 & 0 & 1\end{array}\right]$

Steady state probability distribution difference in day one close value.

$\pi=\left[\begin{array}{llll}0.0057 & 0.4685 & 0.5200 & 0.0057\end{array}\right]$

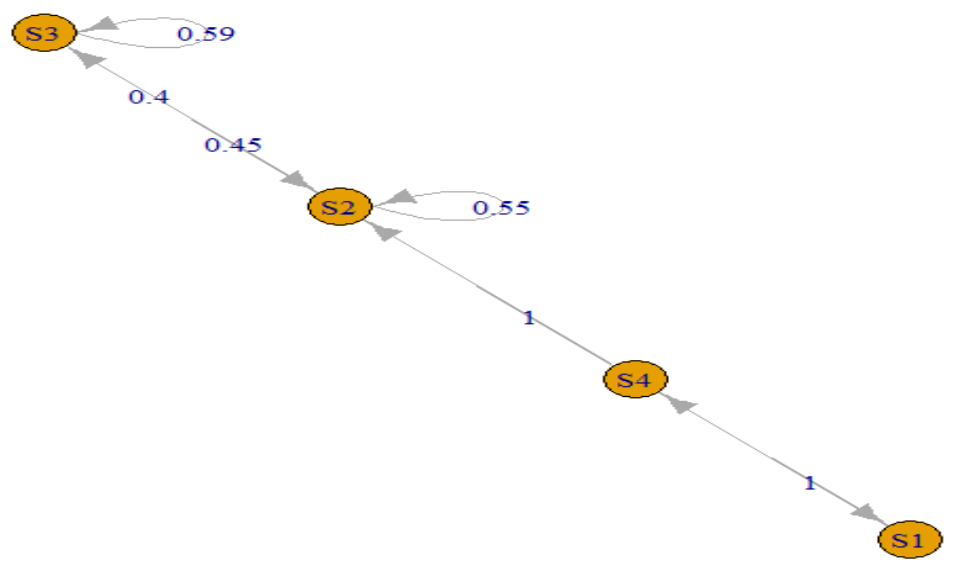

Figure 10. TPM of day one in IOC.NS

Table 12: Transition table with probability values for difference in day two close value.

\begin{tabular}{ccccccccc} 
& S1 & & S2 & & S3 & \multicolumn{3}{c}{ S4 } \\
\hline & I & D & I & D & I & D & I & D \\
\hline S1 & 0 & 0 & 0 & 0 & 0.5 & 0 & 0.5 & 0 \\
\hline S2 & 0 & 0 & 0 & 0.395349 & 0.604651 & 0 & 0 & 0 \\
\hline S3 & 0 & 0.011765 & 0 & 0.611765 & 0.376471 & 0 & 0 & 0 \\
\hline S4 & 0 & 1 & 0 & 0 & 0 & 0 & 0 & 0 \\
\hline
\end{tabular}


Probability values of TPM, EPM, and $\pi$ for difference in day four close value (Figure 11 and Table 12):

$$
\left[\begin{array}{ccccc} 
& S 1 & S 2 & S 3 & S 4 \\
S 1 & 0 & 0 & 0.5 & 0.5 \\
S 2 & 0 & 0.395349 & 0.604651 & 0 \\
S 3 & 0.011765 & 0.611765 & 0.376471 & 0 \\
S 4 & 1 & 0 & 0 & 0
\end{array}\right] \quad \text { and } \quad\left[\begin{array}{ccc} 
& I & D \\
S 1 & 1 & 0 \\
S 2 & 0.604651 & 0.395349 \\
S 3 & 0.376471 & 0.623529 \\
S 4 & 0 & 1
\end{array}\right]
$$

Steady state probability distribution difference in day two close value.

$\pi=\left[\begin{array}{llll}0.0114 & 0.4942 & 0.4885 & 0.0057\end{array}\right]$

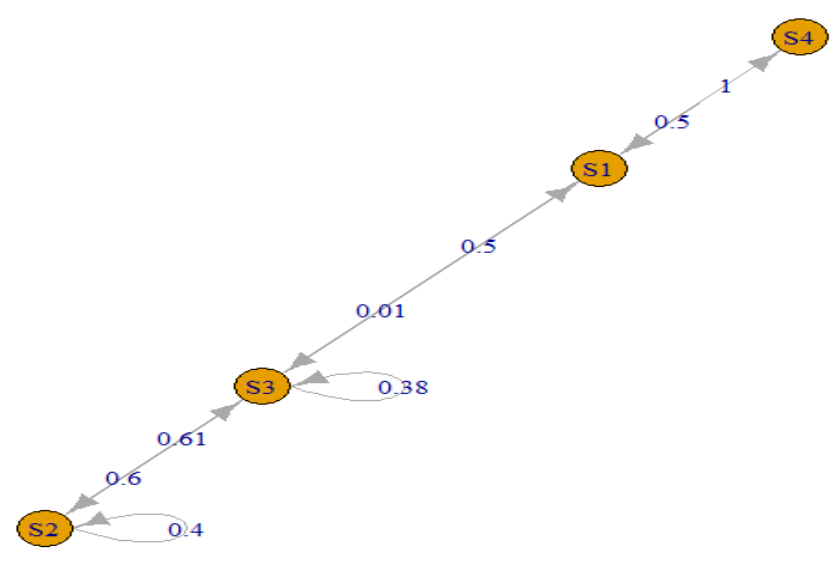

Figure 11. TPM of day two in IOC.NS

Table 13: Transition table with probability values for difference in day three close value.

\begin{tabular}{ccccccccc} 
& S1 & & S2 & & S3 & & S4 & \\
\hline & I & D & I & D & I & D & I & D \\
\hline S1 & 0 & 0 & 0 & 0 & 0 & 0 & 1 & 0 \\
\hline S2 & 0 & 0 & 0 & 0.325301 & 0.674699 & 0 & 0 & 0 \\
\hline S3 & 0 & 0.011628 & 0 & 0.651163 & 0.337209 & 0 & 0 & 0 \\
\hline S4 & 0 & 0.5 & 0 & 0.5 & 0 & 0 & 0 & 0 \\
\hline
\end{tabular}

Probability values of TPM, EPM, and $\pi$ for difference in day four close value (Figure 12 and Table 13):

$$
\left[\begin{array}{ccccc} 
& S 1 & S 2 & S 3 & S 4 \\
S 1 & 0 & 0 & 0 & 1 \\
S 2 & 0 & 0.325301 & 0.674699 & 0 \\
S 3 & 0.011628 & 0.651163 & 0.337209 & 0 \\
S 4 & 0.5 & 0.5 & 0 & 0
\end{array}\right] \quad \text { and } \quad\left[\begin{array}{ccc} 
& I & D \\
S 1 & 1 & 0 \\
S 2 & 0.674699 & 0.325301 \\
S 3 & 0.329412 & 0.662791 \\
S 4 & 0 & 1
\end{array}\right]
$$

Steady state probability distribution difference in day three close value.

$\pi=\left[\begin{array}{llll}0.0114 & 0.4841 & 0.4928 & 0.0114\end{array}\right]$ 


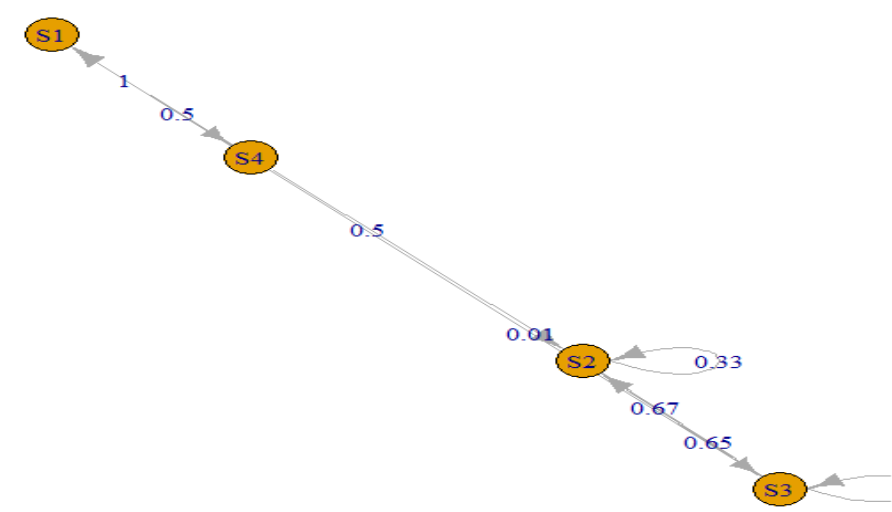

Figure 12. TPM of day three in IOC.NS

Table 14: Transition table with probability values for difference in day four close value

\begin{tabular}{ccccccccc} 
& S1 & & S2 & & S3 & \multicolumn{3}{c}{ S4 } \\
\hline & I & D & I & D & I & D & I & D \\
\hline S1 & 0 & 0 & 0 & 0 & 0.625 & 0 & 0.375 & 0 \\
\hline S2 & 0 & 0 & 0 & 0.333333 & 0.666667 & 0 & 0 & 0 \\
\hline S3 & 0 & 0.0625 & 0 & 0.675 & 0.2625 & 0 & 0 & 0 \\
\hline S4 & 0 & 1 & 0 & 0 & 0 & 0 & 0 & 0 \\
\hline
\end{tabular}

Probability values of TPM, EPM, and $\pi$ for difference in day four close value (Figure 13 and Table 14):

$\left[\begin{array}{ccccc} & S 1 & S 2 & S 3 & S 4 \\ S 1 & 0 & 0 & 0.625 & 0.375 \\ S 2 & 0 & 0.333333 & 0.666667 & 0 \\ S 3 & 0.0625 & 0.675 & 0.2625 & 0 \\ S 4 & 1 & 0 & 0 & 0\end{array}\right] \quad$ and $\quad\left[\begin{array}{ccc} & I & D \\ S 1 & 1 & 0 \\ S 2 & 0.666667 & 0.33333 \\ S 3 & 0.2625 & 0.7375 \\ S 4 & 0 & 1\end{array}\right]$

Steady state probability distribution difference in day four close value.

$\pi=\left[\begin{array}{llll}0.0465 & 0.4709 & 0.4651 & 0.0174\end{array}\right]$

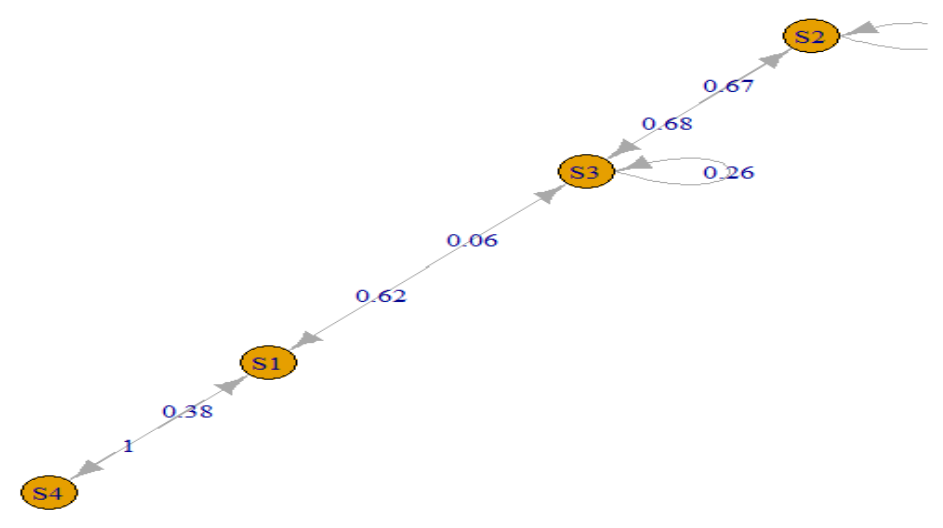

Figure 13. TPM of day four in IOC.NS 
To generate a random sequence of emission symbols and states are calculated by using the function "Hmmgenerate". Murphy(1998) has using the HMM Matlab toolbox syntax is: [Sequence, States] = Hmmgenerate (L, PM, EPM). The length of both sequence and state to be generated is denoted by L. Using the iterative procedure, for each TPM and EPM framed we get an optimum sequence of states generated. The length of the sequence taken as $\mathrm{L}=4$ and the optimum sequence of states obtained from the all four day's differences with TPM and EPM is given in the below and here ' $\pi$ ' is the start symbol.

\section{BPCL.NS}

\begin{tabular}{cclccccccc}
\hline 1. & $\varepsilon$ & $\rightarrow$ & $\mathrm{I}$ & $\rightarrow$ & $\mathrm{D}$ & $\rightarrow$ & $\mathrm{D}$ & $\rightarrow$ & $\mathrm{I}$ \\
& & & $\mathrm{S} 3$ & & $\mathrm{~S} 3$ & & $\mathrm{~S} 2$ & & $\mathrm{~S} 3$ \\
\hline 2. & $\varepsilon$ & $\rightarrow$ & $\mathrm{I}$ & $\rightarrow$ & $\mathrm{I}$ & $\rightarrow$ & $\mathrm{I}$ & $\rightarrow$ & $\mathrm{D}$ \\
& & & $\mathrm{S} 4$ & & $\mathrm{~S} 3$ & & $\mathrm{~S} 2$ & & $\mathrm{~S} 4$ \\
\hline 3. & $\varepsilon$ & $\rightarrow$ & $\mathrm{I}$ & $\rightarrow$ & $\mathrm{D}$ & $\rightarrow$ & $\mathrm{I}$ & $\rightarrow$ & $\mathrm{D}$ \\
& & & $\mathrm{S} 4$ & & $\mathrm{~S} 2$ & & $\mathrm{~S} 3$ & & $\mathrm{~S} 2$ \\
\hline 4. & $\varepsilon$ & $\rightarrow$ & $\mathrm{I}$ & $\rightarrow$ & $\mathrm{D}$ & $\rightarrow$ & $\mathrm{I}$ & $\rightarrow$ & $\mathrm{D}$ \\
& & & $\mathrm{S} 3$ & & $\mathrm{~S} 1$ & & $\mathrm{~S} 2$ & & $\mathrm{~S} 3$ \\
\hline
\end{tabular}

\section{HINDPETRO.NS}

\begin{tabular}{cclccccccc}
\hline 1. & $\varepsilon$ & $\rightarrow$ & $\mathrm{D}$ & $\rightarrow$ & $\mathrm{D}$ & $\rightarrow$ & $\mathrm{D}$ & $\rightarrow$ & $\mathrm{I}$ \\
& & & $\mathrm{S} 2$ & & $\mathrm{~S} 2$ & & $\mathrm{~S} 3$ & & $\mathrm{~S} 3$ \\
\hline 2. & $\varepsilon$ & $\rightarrow$ & $\mathrm{I}$ & $\rightarrow$ & $\mathrm{D}$ & $\rightarrow$ & $\mathrm{D}$ & $\rightarrow$ & $\mathrm{I}$ \\
& & & $\mathrm{S} 3$ & & $\mathrm{~S} 3$ & & $\mathrm{~S} 2$ & & $\mathrm{~S} 3$ \\
\hline 3. & $\varepsilon$ & $\rightarrow$ & $\mathrm{I}$ & $\rightarrow$ & $\mathrm{D}$ & $\rightarrow$ & $\mathrm{I}$ & $\rightarrow$ & $\mathrm{I}$ \\
& & & $\mathrm{S} 4$ & & $\mathrm{~S} 1$ & & $\mathrm{~S} 3$ & & $\mathrm{~S} 2$ \\
\hline 4. & $\varepsilon$ & $\rightarrow$ & $\mathrm{I}$ & $\rightarrow$ & $\mathrm{D}$ & $\rightarrow$ & $\mathrm{I}$ & $\rightarrow$ & $\mathrm{D}$ \\
& & & $\mathrm{S} 4$ & & $\mathrm{~S} 3$ & & $\mathrm{~S} 2$ & & $\mathrm{~S} 2$ \\
\hline
\end{tabular}

\section{$\underline{\text { IOC.NS }}$}

\begin{tabular}{cccccccccc}
\hline 1. & $\varepsilon$ & $\rightarrow$ & $\mathrm{I}$ & $\rightarrow$ & $\mathrm{D}$ & $\rightarrow$ & $\mathrm{I}$ & $\rightarrow$ & $\mathrm{I}$ \\
& & & $\mathrm{S} 4$ & & $\mathrm{~S} 2$ & & $\mathrm{~S} 3$ & & $\mathrm{~S} 2$ \\
\hline 2. & $\varepsilon$ & $\rightarrow$ & $\mathrm{I}$ & $\rightarrow$ & $\mathrm{I}$ & $\rightarrow$ & $\mathrm{I}$ & $\rightarrow$ & $\mathrm{D}$ \\
& & & $\mathrm{S} 3$ & & $\mathrm{~S} 2$ & & $\mathrm{~S} 2$ & & $\mathrm{~S} 2$ \\
\hline 3. & $\varepsilon$ & $\rightarrow$ & $\mathrm{I}$ & $\rightarrow$ & $\mathrm{D}$ & $\rightarrow$ & $\mathrm{I}$ & $\rightarrow$ & $\mathrm{D}$ \\
& & & $\mathrm{S} 4$ & & $\mathrm{~S} 1$ & & $\mathrm{~S} 4$ & & $\mathrm{~S} 1$ \\
\hline 4. & $\varepsilon$ & $\rightarrow$ & $\mathrm{I}$ & $\rightarrow$ & $\mathrm{I}$ & $\rightarrow$ & $\mathrm{D}$ & $\rightarrow$ & $\mathrm{D}$ \\
& & & $\mathrm{S} 3$ & & $\mathrm{~S} 2$ & & $\mathrm{~S} 2$ & & $\mathrm{~S} 3$ \\
\hline
\end{tabular}

\section{Conclusion}

Stock market prediction is challenging and interesting due to its randomness. Hidden Markov Model can be used for Stock market assumption by finding stowed away models. Here the Hidden Markov model easily identified such as four states of the stock market and also it was used to predict the future values. The most elevated worth in the Optimum State Sequences is the better exhibition of the specific Sequence. 
Hidden states and successions have been produced to effectively distinguish the level of the arrangement whether the following day values is increasing. And furthermore distinguished whether the increasing level is moderate high or high or very high and furthermore diminishing level whether moderate low or low or very low. This model will be particularly helpful for present moment just as long haul financial backers.

\section{Reference}

1. Ali Sasani and Stephanie Tibado (2020). Stock Market Prediction using Hidden Markov Model and Neural Network, International Journal of Engineering and Applied Sciences, 7(4), 5-10.

2. Deneshkumar V, Senthamarai Kannan K and N. Sonai Muthu (2020). Stock Market Trend Prediction Using Hidden Markov Model, Forecasting in Mathematics - Recent Advances, New Perspectives and Applications, 1-12.

3. Gupta A and B. Dhingra (2012). Stock Market prediction using Hidden Markov Models, Engineering and Systems IEEE Students Conference, 1-4.

4. Hassan M R and B. Nath (2005). Stock Market Forecasting Using Hidden Markov Model: A New Approach, Proceedings of the 5th International Conference on Intelligent Systems Design and Applications, 192-196.

5. Jyoti Badge (2012). Forecasting of Indian Stock Market by Effective Macro- Economic Factors and Stochastic Model, Journal of Statistical and Econometric Methods, 1 (2), 39-51.

6. Kavitha G, Udhayakumar A and D. Nagarajan (2013). Stock Market Trend Analysis Using Hidden Markov Models, International Journal of Computer Science and Information Security, 11(10), 103-110.

7. Mohamed Ashik. A and K. Senthamarai Kannan (2017). Forecasting National Stock Price Using ARIMA Model, Global and Stochastic Analysis, 4(1), 77-81.

8. Murphy K (1998). HMM toolbox for Matlab. Internet: http://www.cs.ubc. $\mathrm{ca} /$ murphyk/Software/HMM/hmm.

9. Nguyet Nguyen (2018). Hidden Markov Model for Stock Trading, International Journal of Financial Studies, 6(2), 1-17.

10. Rabiner LR. (1989). A Tutorial on Hidden Markov Models and Selected Applications in Speech Recognition. Proceedings of the IEEE, February 77(2), 257-286.

11. Rabiner. L (1993). Theory and Implementation of Hidden Markov Models. Fundamentals of Speech Recognition. 
12. Sasikumar R and A. Sheik Abdullah (2015). Applications of Various Stochastic Models in Financial Prediction, International Journal of Scientific and Innovative Mathematical Research, 3(3), 852-857.

13. Sasikumar R and A. Sheik Abdullah (2016). Forecasting the Stock Market Values Using Hidden Markov Model, International Journal of Business Analytics and Intelligence, 4(1), 17-21. 